\title{
Chronic Chemogenetic Stimulation of the Nucleus Accumbens Produces Lasting Reductions in Binge Drinking and Ameliorates Alcohol-Related Morphological and Transcriptional Changes
}

\author{
Dar'ya Y. Pozhidayeva ${ }^{1,2,3}$, Sean P. Farris ${ }^{4}$, Calla M. Goeke ${ }^{1,2}$, Evan J. Firsick ${ }^{2}$, \\ Kayla G. Townsley ${ }^{1,2}{ }^{-}$, Marina Guizzetti ${ }^{1,2}$ and Angela R. Ozburn ${ }^{1,2, *}$ \\ 1 Department of Behavioral Neuroscience, Oregon Health \& Science University, Portland, OR 97239, USA; \\ pozhiday@ohsu.edu (D.Y.P.); calliegoeke@gmail.com (C.M.G.); kaala96@gmail.com (K.G.T.); \\ guizzett@ohsu.edu (M.G.) \\ 2 Research \& Development, VA Portland Health Care System, Portland, OR 97239, USA; firsick@ohsu.edu \\ 3 Chemistry Department, Portland State University, Portland, OR 97207, USA \\ 4 College of Natural Sciences, Waggoner Center for Alcohol and Addiction Research, University of Texas at \\ Austin, Austin TX 78712, USA; spfarris@utexas.edu \\ * Correspondence: ozburn@ohsu.edu; Tel.: +1-503-220-8262 (ext. 58393); Fax: +1-503-721-1029
}

Received: 17 December 2019; Accepted: 17 February 2020; Published: 18 February 2020

\begin{abstract}
Binge drinking is a dangerous pattern of behavior. We tested whether chronically manipulating nucleus accumbens (NAc) activity (via clozapine-N-oxide (CNO) and Designer Receptors Exclusively Activated by Designer Drugs (DREADD)) could produce lasting effects on ethanol binge-like drinking in mice selectively bred to drink to intoxication. We found chronically increasing NAc activity (4 weeks, via CNO and the excitatory DREADD, hM3Dq) decreased binge-like drinking, but did not observe CNO-induced changes in drinking with the inhibitory DREADD, hM4Di. The CNO/hM3Dq-induced reduction in ethanol drinking persisted for at least one week, suggesting adaptive neuroplasticity via transcriptional and epigenetic mechanisms. Therefore, we defined this plasticity at the morphological and transcriptomic levels. We found that chronic binge drinking (6 weeks) altered neuronal morphology in the NAc, an effect that was ameliorated with $\mathrm{CNO} / \mathrm{hM} 3 \mathrm{Dq}$. Moreover, we detected significant changes in expression of several plasticity-related genes with binge drinking that were ameliorated with CNO treatment (e.g., Hdac4). Lastly, we found that LMK235, an HDAC4/5 inhibitor, reduced binge-like drinking. Thus, we were able to target specific molecular pathways using pharmacology to mimic the behavioral effects of DREADDs.
\end{abstract}

Keywords: binge drinking; genes; signaling pathways; DREADDs or chemogenetics; high drinking in the dark mice

\section{Introduction}

Binge drinking is a problematic pattern of behavior and often leads to the development of alcohol use disorders (AUD; https://niaaa.scienceblog.com/24/niaaa-scientists-provide-more-evidence-thatbinge-drinking-may-indicate-vulnerability-to-alcohol-use-disorder/; 02/15/20). Binge drinking is defined by the National Institute on Alcohol Abuse and Alcoholism as having 4 to 5 drinks within $2 \mathrm{~h}$ and/or achieving a blood alcohol level (BAL) $>80 \mathrm{mg} \%$ (https://www.niaaa.nih.gov/alcohol-health/ overview-alcohol-consumption/moderate-binge-drinking; 02/15/20). Limited access ethanol drinking paradigms are used to model binge/intoxication in animal models (however, not all limited access paradigms and animal strains drink to intoxication). In the limited access Drinking in the Dark 
(DID) paradigm, mice are offered an ethanol solution early into the active period of their circadian cycle and can achieve BALs $>80 \mathrm{mg} \%$, suggesting they drink to intoxication [1]. C57BL/6J mice are typically reported as high drinking and can achieve BALs $>80 \mathrm{mg} \%$ in the DID paradigm; however, significant inbred strain differences have been observed suggesting there exists a genetic contribution to this phenotype [2,3]. The DID assay was used to independently create two lines of mice, HDID-1 and HDID-2, that were selectively bred (from genetically heterogeneous HS/Npt progenitors) for high BALs after DID [4,5]. HDID and HS/Npt mice have been extensively characterized and HDID mice represent a unique genetic animal model of risk for drinking to intoxication [6-14]. FDA approved compounds for treatment of AUD, as well as several investigational compounds, have been tested for efficacy in reducing binge-like drinking using the DID paradigm in C57BL/6J and HDID mice, as well as other genotypes, with mixed results [15-20]. Together, the results of these studies suggest that testing potential therapies in more than one inbred strain may represent a more beneficial strategy for clinical translation.

Koob and Volkow (2010) reviewed decades of clinical and pre-clinical studies to address the neural circuitry associated with the three stages of the addiction cycle: binge/intoxication, withdrawal/negative affect, and preoccupation/anticipation [21]. The nucleus accumbens (NAc) is identified as an important region for each of these three stages. There is also extensive evidence that altering activity in the NAc reduces alcohol drinking, craving, and relapse. To achieve this, clinical studies have applied deep brain stimulation (DBS) to the NAc in humans [22-24]. Human studies were carried out in males with treatment resistant alcohol use disorder (AUD), where NAc DBS of the shell relieved symptoms of craving and reduced relapse [22]. Pre-clinical studies have also found that DBS of the NAc reduced alcohol intake that was generalizable to different strains of rats and drinking paradigms $[25,26]$. Many of these studies report that DBS of either the NAc core or shell (or both) are effective in reducing drug-related behaviors. Although DBS of the NAc decreases alcohol drinking and craving, its effects are not lasting (reviewed in [27]).

The promising results of NAc DBS studies led us to test whether DREADDs (Designer Receptors Exclusively Activated by Designer Drugs) could be used to alter neuronal activity in the NAc and whether they could alter binge-like drinking. DREADDs (developed and characterized by Dr. Bryan Roth and his research team) have been an important tool for neuroscience research, enabling identification of brain circuitry involved in specific behaviors [28]. Here, we tested the effects of clozapine-N-oxide (CNO) on binge drinking in mice expressing excitatory DREADDs (hM3Dq), inhibitory DREADDs (hM4Di), or control (GFP) in the nucleus accumbens core. We tested the effects of CNO/DREADDs in female mice from a high drinking mouse line (HDID-1; where $>90 \%$ of mice drink to intoxication) and hypothesized that $\mathrm{CNO} / \mathrm{hM} 3 \mathrm{Dq}$ would decrease binge-like drinking. DREADDs are mutagenized G-protein coupled receptors (GPCRs), and since we know GPCRs play an important role in neuroplasticity, we also tested the hypothesis that chronic stimulation of DREADDs (4 weeks) could lead to lasting reductions in binge-like drinking. We hypothesized that this behavioral plasticity could be observed at cellular and molecular levels via lasting changes in neuronal morphology and the transcriptome. Here, we focused on identifying genes and biological pathways that were altered with chronic binge-drinking ( 6 weeks) and ameliorated with chronic CNO treatment (4 weeks). We then pharmacologically targeted these biological pathways in male and female HDID-1 mice to determine whether they reduced binge like drinking.

\section{Materials and Methods}

\subsection{Experimental Animals}

Experimental Animals: Adult High Drinking in the Dark line 1 (HDID-1) mice (generously provided by Dr. John Crabbe, Portland, Oregon, USA) aged 2-5 months were used for all experiments in this study. HDID-1 mice are a genetic model of risk for drinking to intoxication and were selectively bred from a genetically heterogeneous stock, $\mathrm{HS} / \mathrm{Npt}[4,5]$. Mice were bred and maintained in a 
reversed 12/12-h light/dark cycle, with lights off at 08:30 and lights on at 20:30. Cages were made of polycarbonate $(28 \times 17 \times 11.5 \mathrm{~cm})$ containing about $2.5 \mathrm{~cm}$ of cob bedding. Purina brand food (5LOD, PMI Nutrition International, Brentwood, MO, USA) was suspended in a wire-top, and food and water were available ad libitum. Female mice were used in experiments 1 and 2 to facilitate comparison with data our group had previously published in C57BL/6J mice [29]. Male and female mice were used in experiment 3. All procedures in this study were performed in accordance with NIH Guidelines for the Care and Use of Laboratory Animals and were approved by the VA Portland Health Care System's Institutional Animal Care and Use Committee.

\subsection{Drinking in the Dark (DID)}

Binge-like drinking of ethanol in mice was assessed using Drinking in the Dark (using a variation of Rhodes et al., 2005 as described below). All mice had daily access to 20\% ethanol (v/v, in tap water; Decon Laboratories, Inc., PA, USA) for $2 \mathrm{~h}$ (offered three hours into lights off).

\subsection{Drugs}

For experiments 1 and 2: 30 min prior to DID, mice received IP (intraperitoneal) injections of either VEH (vehicle: 1\% DMSO (Hybri-Max, Sigma Life Sciences, MO, USA) in saline $(0.9 \% \mathrm{NaCl}$; Baxter International, IL, USA)), or $1 \mathrm{mg} / \mathrm{kg} \mathrm{CNO} \mathrm{(1 \%} \mathrm{DMSO} \mathrm{in} \mathrm{saline;} \mathrm{RTI} \mathrm{International,} \mathrm{NC,} \mathrm{USA).}$ For experiment 3, 60 min prior to DID, mice were injected IP with either vehicle (5\% DMSO in Dulbecco's phosphate buffered saline; Gibco, Sigma Life Sciences, MO, USA) or 5 or 20 mg/kg LMK 235 (Selleck Chemicals, LLC, TX, USA).

\subsection{Experiment 1}

Female HDID-1 mice (S32.G34) were anesthetized with a mixture of ketamine (125 mg/kg) and xylazine $(12.5 \mathrm{mg} / \mathrm{kg})$ in saline and received bilateral stereotaxic infusions of $1 \mu \mathrm{L}$ purified high titer AAV into the NAc (rAAV2-hSyn-eGFP (GFP; $n=15$; control), rAAV2-hSyn-HA-hM3D(Gq)-IRES-mCitrine (hM3Dq; $n=13$; excitatory DREADD), or rAAV2-hSyn-HA-hM4D(Gi)-IRES-mCitrine (hM4Di; $n=8$; inhibitory DREADD)). Injections were administered to the following coordinates targeting the NAc core (from bregma, in $\mathrm{mm}$ ): angle $10^{\circ}, \mathrm{AP}+1.5$, Lat $+1.7, \mathrm{DV}-4.6$ and $-4.0 ; 0.5 \mu \mathrm{L}$ delivered at $\mathrm{DV}-4.6$ and $0.5 \mu \mathrm{L}$ delivered at DV -4.0. AAV titers were $2-3.5 \times 10^{12} \mathrm{vg}$ and were purchased from the University of North Carolina Viral Vector Core. Two weeks later, mice were individually housed and habituated to novel sipper tubes for one week. The DID experiment was then carried out daily for 6 weeks. Mice were serially treated with vehicle prior to DID during week 1 to establish baseline drinking, CNO during weeks 2-5 to measure the effects of chronic treatment, and then mice were treated with vehicle again during week 6 to determine if there were any lasting effects of chronic CNO treatment. The serial treatment is represented in the figures as $\mathrm{VEH} / \mathrm{CNO} / \mathrm{VEH}$. Ethanol intake was measured as described in Purohit et al., 2018 [29]. At the completion of this study, mice were deeply anesthetized and transcardially perfused with 0.01 M PBS and 4\% $w / v$ paraformaldehyde (PFA). Brains were extracted, sectioned at $30 \mu \mathrm{m}$ using a freezing stage microtome (Model No. 860, American Optical Corp., Buffalo, New York, NY, USA), and processed for immunofluorescence to verify injection placements. Immunohistochemical staining was performed following standard procedures using rabbit anti-GFP or anti-HA primary antibody (anti-GFP, 1:20,000 dilution: Catalog\#ab290, AbCam, Cambridge, MA, USA; anti-HA, 1:1000 dilution: Catalog \#3724S, Cell Signaling Technologies, Danvers, MA, USA) and goat anti-rabbit Alexa 488 secondary antibody (1:500 dilution: Catalog \#A-11088, Thermofisher Scientific, Waltham, MA, USA). Brain sections were mounted on microscope slides (Catalog \#12-550-15, Fisherbrand, Waltham, MA, USA) using Vectashield Antifade Mounting Medium with DAPI (Vector Laboratories, Burlingame, CA, USA) and observed using an Olympus BX60 fluorescence microscope (Olympus, Center Valley, PA, USA). Animals were excluded if expression was not localized to the NAc (hM4Di $n=2$, GFP $n=3$ ). 


\subsection{Experiment 2}

Female HDID-1 mice (S35.G37) were stereotaxically injected with $0.5 \mathrm{uL}$ rAAV2/5-CMV-Cre-GFP and $0.5 \mathrm{uL}$ rAAV2-hSyn-DIO-hM3Dq-mCherry bilaterally into the NAc targeting the NAc core/shell border region (from bregma, in mm: angle $10^{\circ}, \mathrm{AP}+1.34$, Lat +1.5 , DV -4.5). AAV titers were 4-8 x 10 12 vg and were purchased from the University of North Carolina Viral Vector Core (Cre) and Add Gene (DIO constructs). Two weeks later, mice were individually housed and habituated to novel sipper tubes for one week prior to carrying out a 6 week (7 days/week) DID experiment (as described for experiment (1). There were four groups of mice: (a) mice treated with vehicle that only had access to water (control group), (b) mice treated with $\mathrm{VEH} / \mathrm{CNO} / \mathrm{VEH}$ that only had access to water (to test the effects of $\mathrm{CNO}$ on neuronal morphology and the transcriptome), (c) mice treated with vehicle that had access to ethanol (to test the effects of chronic binge-like drinking on morphology and the transcriptome), (d) mice treated with $\mathrm{VEH} / \mathrm{CNO} / \mathrm{VEH}$ that had access to ethanol (to test the effects of CNO and chronic binge-like drinking on neuronal morphology and the transcriptome). Mice were serially treated with vehicle prior to DID during week 1 to establish baseline drinking, then vehicle or CNO during weeks 2-5 to measure the effects of chronic treatment, and then mice were treated with vehicle again during week 6 to determine whether there were any lasting effects of chronic CNO treatment. Ethanol intake was measured as described in Purohit at al., 2018 [29]. For neuronal morphology analysis, female HDID-1 mice (S34.G36; $n=4-5 /$ fluid type/treatment) were deeply anesthetized $22 \mathrm{~h}$ after the last DID and transcardially perfused with $0.01 \mathrm{M}$ PBS and $4 \% w / v$ paraformaldehyde (PFA). Brains were extracted and placed in $4 \%$ PFA for $24 \mathrm{hr}$ at $4{ }^{\circ} \mathrm{C}$ before processing for Golgi-Cox staining as described in Bayram-Weston et al., 2016 [30]. After 14 days at room temperature and low ambient light, the tissue was transferred into a $30 \%$ w/v sucrose solution for $24-72 \mathrm{hr}$ (low light at $4{ }^{\circ} \mathrm{C}$ ), prior to sectioning and mounting. Brains were sectioned coronally using a Compresstome at $200 \mu \mathrm{m}$ (Model VF 300-0Z Microtome with AutoZero Z technique, Precisionary, Greenville, NC, USA) and mounted on gelatin-coated slides. Slides were prepared according to Section 2.3 of Bayram-Weston et al., 2016 [30], and cover-slipped with Permount ${ }^{\mathrm{TM}}$ Mounting Medium (Fisher Scientific Co., Waltham, MA, USA). For transcriptomic analysis, mice ( $n=11-12 /$ fluid type/treatment) were euthanized $22 \mathrm{~h}$ after the last DID session via cervical dislocation and rapid decapitation. Whole brains were frozen on powdered dry ice, $300 \mu \mathrm{m}$ coronal sections were collected using a cryostat, and $1.0 \mathrm{~mm}$ frozen tissue punches were collected from the NAc and processed for RNA Sequencing.

Microscopy: NAc medium spiny neurons were traced blind to condition using the software Neurolucida (Version 11, MBF Bioscience, Williston, VT, USA) on a Leica DM500b microscope using a DFC36 FX camera. Parameters analyzed were: complexity (defined as (sum of the terminal orders + the number of terminals) $\times$ (total dendritic length/number of dendrites)), average dendrite length $(\mu \mathrm{m})$, number of nodes, number of ends, total dendritic length $(\mu \mathrm{m})$, sum of the terminal orders (defined as number of sister branches encountered when tracing a dendrite from the tip back to the cell body), dendrite quantity, and branch sum (defined as the total number of segments between nodes). Neuronal complexity is a measure automatically derived from Neurolucida. In addition, Sholl analysis was carried out. The parameters analyzed in each $10 \mu \mathrm{m}$ radius were: number of intersections, length $(\mu \mathrm{m})$, number of nodes, number of ends, and the area under the curve was also calculated. The number of animals and cells per group that were included in analyses are given in Table 1.

RNA Sequencing and Validation of viral gene transduction: NAc tissue punches were mechanically homogenized in PureZol and RNA was isolated using the Aurum Total RNA Fatty and Fibrous Tissue kit (Bio-Rad Laboratories, Inc., Hercules, CA, USA). RNA (100 ng) was processed to cDNA using the BioRad iScript cDNA synthesis kit (Catalog \#: 1708890; Bio-Rad Laboratories, Inc., Hercules, CA, USA) according to the manufacturer's protocol. Quantitative real-time PCR was used to measure expression of mCherry to confirm viral expression of hM3Dq-mCherry in samples. A PrimePCR custom assay for mCherry (FOR: 5'-AGCGCGTGATGAACTTCGA-3' REV: 5'-CGCAGCTTCACCTTGTAGATGA-3'; PROBE: 5'-CCGTGACCCAGGACTC; 5' 6-FAM, 3' Iowa Black FQ) and Rps18 (Mouse, FAM, qMmuCEP0053856) probes were used (Bio-Rad Laboratories, Inc., Hercules, CA, USA). mCherry 
expression levels (relative to Rps18) were verified as detectable and similar for all samples (data not shown). RNA samples were sent to the Massively Parallel Sequencing Shared Resource (MPSSR) at Oregon Health \& Science University for quality assessment (via 2100 BioAnalyzer, Agilent Technologies, Palo Alto, CA, USA), library preparation, and sequencing. The library was prepared using the Illumina TruSeq RNA-Seq Library Protocol. Sequencing was executed via an Illumina HiSeq 2500 Sequencer (Illumina, San Diego, CA, USA) with poly(A)+ stranded selection and paired-end reads at 50 cycles.

Table 1. The number of animals and cells per group that were included in morphological analyses are provided here.

\begin{tabular}{|c|c|c|c|c|c|c|c|}
\hline \multicolumn{8}{|c|}{ Number of Animals and Cells Per Group Included for Morphological Analyses } \\
\hline Group & Animal (n) & Cells/Brain\#1 & Cells/Brain\#2 & Cells/Brain\#3 & Cells/Brain\#4 & Cells/Brain\#5 & Total Cells \\
\hline $\mathrm{H}_{2} \mathrm{O}+\mathrm{Veh}$ & 4 & 9 & 5 & 6 & 9 & 0 & 29 \\
\hline $\mathrm{H}_{2} \mathrm{O}+\mathrm{CNO}$ & 5 & 5 & 7 & 5 & 6 & 13 & 36 \\
\hline $\mathrm{EtOH}+\mathrm{Veh}$ & 4 & 6 & 10 & 5 & 8 & 0 & 29 \\
\hline $\mathrm{EtOH}+\mathrm{CNO}$ & 4 & 6 & 5 & 6 & 6 & 0 & 23 \\
\hline Totals & 17 & & & & & & 117 \\
\hline
\end{tabular}

\subsection{Experiment 3}

Male and female HDID-1 mice (S38.G40, S39.G41, S41.G43) were individually housed and habituated to novel sipper tubes one week prior to DID. Mice were tested four days per week (as in [1]). Mice were serially treated with vehicle prior to DID (during weeks 1 or 1 and 2) to establish baseline drinking, and then 0,5 , or $20 \mathrm{mg} / \mathrm{kg}$ LMK 235 over the next 2 weeks to measure the effects of chronic treatment with this HDAC4/5 inhibitor. Ethanol intake was measured using volumetric tubes in $2 \mathrm{~h}$ intervals on all 4 days [8].

\subsection{Data Analysis and Statistics}

\subsubsection{Behavior}

For experiments 1 and 2, daily ethanol intake data was averaged for each mouse for each treatment period. Intake data were then analyzed using a repeated measures one-way analysis of variance (ANOVA) to determine whether there was an effect of treatment period on drinking. For significant results $(p<0.05)$, post-hoc tests were carried out to compare baseline drinking to clozapine-n-oxide (CNO) treatment and washout periods. For experiment 3, drinking data for each $2 \mathrm{~h}$ interval was averaged for each period (baseline vs. treatment weeks). Data was analyzed using a two-way repeated measures ANOVA (dose $\times$ treatment period).

\subsubsection{Morphology}

As the data was nested, with multiple neurons within a brain (dependent) being compared to multiple neurons from other brains (independent), we used a linear mixed effect analysis [31]. We did this using $\mathrm{R}$ and the package lme4 to perform a multilevel analysis [32]. The animal was accounted for as a random effect, due to multiple cells for each animal being examined. We used the fluid type [ethanol $(\mathrm{EtOH})$ vs. water $\left(\mathrm{H}_{2} \mathrm{O}\right)$ ] and the treatment (CNO vs. Vehicle) as fixed effects, and $p$-values were obtained by likelihood ratio tests. For all morphometric parameters, we examined the main effect of both variables, as well as their interaction. We then corrected for multiple comparisons using the Benjamini-Hochberg approach to adjust the nominal p-values to the q-values of the False Discovery Rate (FDR) [33]. Sholl analyses were analyzed by radius as well as area under the curve (AUC). $p$-values and $q$-values less than 0.05 were considered significant, and data was presented as mean \pm standard error of the mean (SEM). 


\subsubsection{Transcriptomic Analysis}

Raw FASTA files generated from sequencing were aligned to the Genome Reference Consortium Mouse Reference 38, based on the Mus musculus strain C57BL/6J (GRCm38; also known as mm10). Basecall quality was assessed using FastQC [34] and alignment was run using STAR: ultrafast universal RNA-seq aligner [35] (version: 2.6.0) where paired-end reads were specified, the maximum number of mismatches per pair was indicated as 3 and maximum number of multiple alignments allowed per read was indicated as 1. Count data was generated using HTSeq (version 0.9.1) against the Encode vM4 annotation with paired-end read order specification [36]. After filtering, normalization and differential expression were performed using Bioconductor (version 3.34.9) in R [37]. The Limma voom function was utilized to perform empirical Bayes-moderated t-statistics to identify Differentially Expressed Genes (DEGs, $p<0.05$ ) between fluid and treatment groups [38]. Mean-variance relationships and observational-level weights were calculated from log2-counts per million (log-CPM). Gene expression overlap was identified using BioVenn [39]. Expression patterns for genes mediating morphology were based on comparisons made during morphological analysis. Groups compared included only $\mathrm{EtOH}(\mathrm{VEH})$ vs. $\mathrm{H}_{2} \mathrm{O}(\mathrm{VEH})$ and $\mathrm{EtOH}(\mathrm{CNO})$ vs. $\mathrm{H}_{2} \mathrm{O}(\mathrm{CNO})$. Target genes identified for comparison were based on literature by Russo et al., 2010, Arikkath et al., 2012 and Uys et al., 2016 [40-42]. Weighted gene co-expression network analysis (WGCNA; version 1.67) was performed using R implementation [43]. A signed, hybrid co-expression network was constructed covering 24,421 genes meeting the cut-off criterion of 1 CPM. Prior to network construction, $10 \%$ of genes exhibiting the lowest correlation values were removed via SUMCOR as in Tritchler et al., 2009 [44]. The co-expression network was constructed via Pearson correlation between all gene pairs and the exponentiation of the resulting absolute value to a power beta $=7$. A consensus network was constructed utilizing samples from all sequenced RNA samples. Modules were detected in each network via hierarchical clustering where average linkage and the WGCNA cuttreeHybrid function was used with the following parameters: cutHeight $=0.998$, minClusterSize $=100$, and deepSplit $=0$. Total network connectivity (degree) of each gene was calculated via the sum total of all edges in the network, where modular connectivity restricted the edges included to the gene's own module. Pseudo and Riken genes exhibiting abnormally high connectivity were sequestered and removed. Module quality was assessed using the WGCNA modulePreservation function. The geneMania and GOrilla enrichment algorithms were used to identify gene ontology annotation and enrichment for biological pathways $[45,46]$. Ontology for transcription factors was performed using the enrichR algorithm [47]. All code used is accessible via https://github.com/Pozhidayevad/Ozburn_RNA-Seq_Analyses. RNA Seq data is accessible via GEO (accession number GSE).

\section{Results}

\subsection{Experiment 1: Chronically Increasing Gq Signaling in the NAc Produces Lasting Reductions in Binge-Like Drinking}

To identify the effect of manipulating NAc activity (via CNO and hM3Dq- or hM4Di-DREADDs) on binge-like ethanol drinking behavior, HDID-1 mice were subjected to the DID paradigm. The effects of treatment (via serial administration of vehicle, $\mathrm{CNO}$, and vehicle) on ethanol binge-like drinking in mice expressing GFP (Figure 1A), hM4Di (Figure 1B), and hM3Dq (Figure 1C) is presented as the mean $( \pm$ SEM) ethanol intake $(\mathrm{g} / \mathrm{kg} / 2 \mathrm{~h})$ across each treatment period. Mice expressing GFP or hM4Di in the NAc did not exhibit significant changes in ethanol intake during CNO treatment or vehicle washout periods (relative to baseline intake). However, mice expressing hM3Dq in the NAc exhibited a significant reduction in ethanol intake during chronic CNO treatment and during the vehicle washout periods relative to vehicle baseline intake (one way ANOVA- $\mathrm{F}(2,35)=4.84$, $p<0.01$; post-hoc-VEH(baseline) vs. CNO, $p<0.05$; VEH (baseline) vs. VEH (washout), $p<$ 0.05). Thus, chronically increasing Gq signaling in the NAc produced lasting effects on binge-like drinking behaviors. 
A

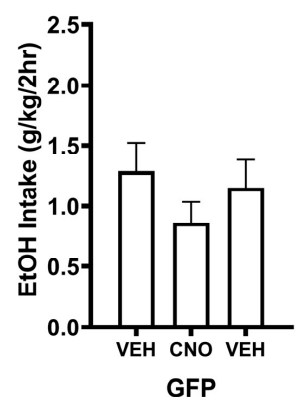

B

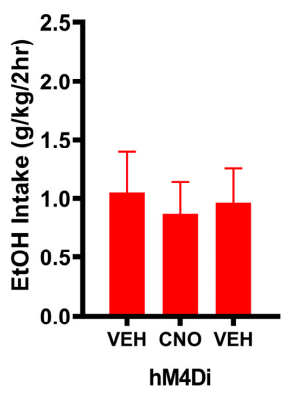

C

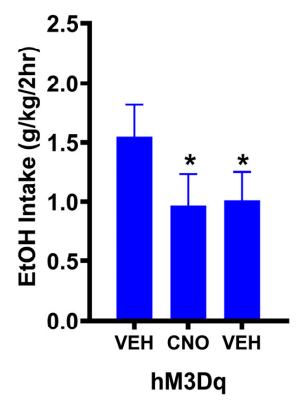

D

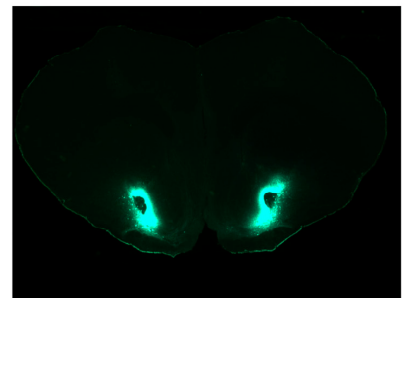

Figure 1. Chronically increasing Gq signaling in the NAc produced lasting reductions in binge-like drinking. Mean (+/- SEM) 2-hr ethanol (EtOH) intake during drinking in the dark (DID) is shown as a function of the three treatment periods for each group as follows: (A) control (GFP), (B) inhibitory DREADDs (hM4Di), and (C) mice expressing excitatory DREADDs (hM3Dq). The first bar is data from week 1 to measure baseline intake in response to vehicle, the second bar is data from weeks 2-5 to measure intake in response to chronic vehicle (VEH) (A) or clozapine-N-oxide (CNO) (B), and the third bar is data from week 6 to measure intake in response to $\mathrm{VEH}$ again (and determine whether there were lasting effects of $\mathrm{CNO}$ ). (C) Significant decreases were observed only in hM3Dq during $\mathrm{CNO}$ treatment and washout, relative to baseline intake (one way ANOVA- $\mathrm{F}(2,35)=4.84, p<$ 0.05; post-hoc-VEH(baseline) vs. CNO, ${ }^{*} p<0.05$; VEH (baseline) vs. VEH (washout), ${ }^{*} p<0.05$ ). No significant differences in intake over treatment periods were observed for GFP or hM4Di groups. (D) $2 \times$ image of coronal section showing targeting of NAc core in a representative mouse from this study (where green indicates viral transduction).

3.2. Experiment 2: Chronically Increasing Gq Signaling in the NAc Produces Lasting Reductions in Binge-Like Drinking, the Transcriptome, and Neuronal Morphology

\subsubsection{Behavioral Results}

Results from experiment 1 showed that chronic stimulation using hM3Dq excitatory DREADDs produced a robust and lasting reduction in binge-like drinking in a model of genetic risk for binge-like drinking, thus the previous study was replicated using hM3Dq DREADDs exclusively. Here, the results of chronic CNO administration in mice hM3Dq excitatory DREADDs in the NAc were similar to data from experiment 1 . Figure $2 \mathrm{~A}$ shows ethanol drinking in response to chronic treatment with vehicle throughout the duration of the experiment (open bar). The EtOH(VEH) group experienced no significant changes at any point of the experiment in average ethanol intake $(\mathrm{g} / \mathrm{kg} / 2 \mathrm{~h}$ DID session) relative to baseline intake. Conversely, the group treated with $\mathrm{CNO}$ shown in Figure 2B experienced significant and lasting reductions in ethanol intake during CNO treatment (one way ANOVA-F $(2,35)$ $=10.49, p<0.01$; Dunnett post-hoc test revealed significant differences for VEH baseline vs. CNO ( $p$ $<0.01)$ and VEH baseline vs. VEH washout $(p<0.05))$. Replication of this finding provides strong evidence for behavioral plasticity. We next sought to identify how this plasticity manifested on a morphological and transcriptomic level.

\subsubsection{Morphological Results}

Behavioral plasticity has morphological underpinnings, which can be observed and analyzed. Therefore, we measured medium spiny neurons in the NAc for a variety of morphometric parameters, in order to determine the effects of treatment ( $\mathrm{CNO}$ or vehicle), fluid type $\left(\mathrm{EtOH}\right.$ or $\left.\mathrm{H}_{2} \mathrm{O}\right)$, or whether there was an interaction between the two treatments. We analyzed neuronal complexity, determined via the equation (sum of terminal orders + number of terminals) $\times$ (total dendritic length/number of dendrites) (Figure 3A), length per dendrite (Figure 3B), number of nodes (Figure 3C), number of ends (Figure 3D), total dendritic length per neuron (Figure 3E), the sum of terminal orders, which was defined as the number of sister branches encountered when tracing a dendrite from the tip back to the 
cell body (Figure 3F), the quantity of dendrites (Figure 3G), and the branch sum, defined as the total number of segments between nodes (Figure 3H). In most parameters, there was an increase following exposure to EtOH (statistics for analyses are listed in Supplementary Table S5). This main effect was significant for neuronal complexity, the sum of terminal orders, and the branch sum, approached significance following corrections for multiple comparisons in the length per dendrite and the number of ends, and was significant before corrections for multiple comparisons in the number of nodes (Supplementary Table S5). There were no significant changes in the total dendritic length or the number of dendrites. There was also no effect of the injection (CNO or Vehicle), nor was there an interaction between the injection and exposure conditions. However, it is worth noting that the effects observed for the $\mathrm{EtOH}(\mathrm{VEH})$ group were not observed in the $\mathrm{EtOH}(\mathrm{CNO})$ group.
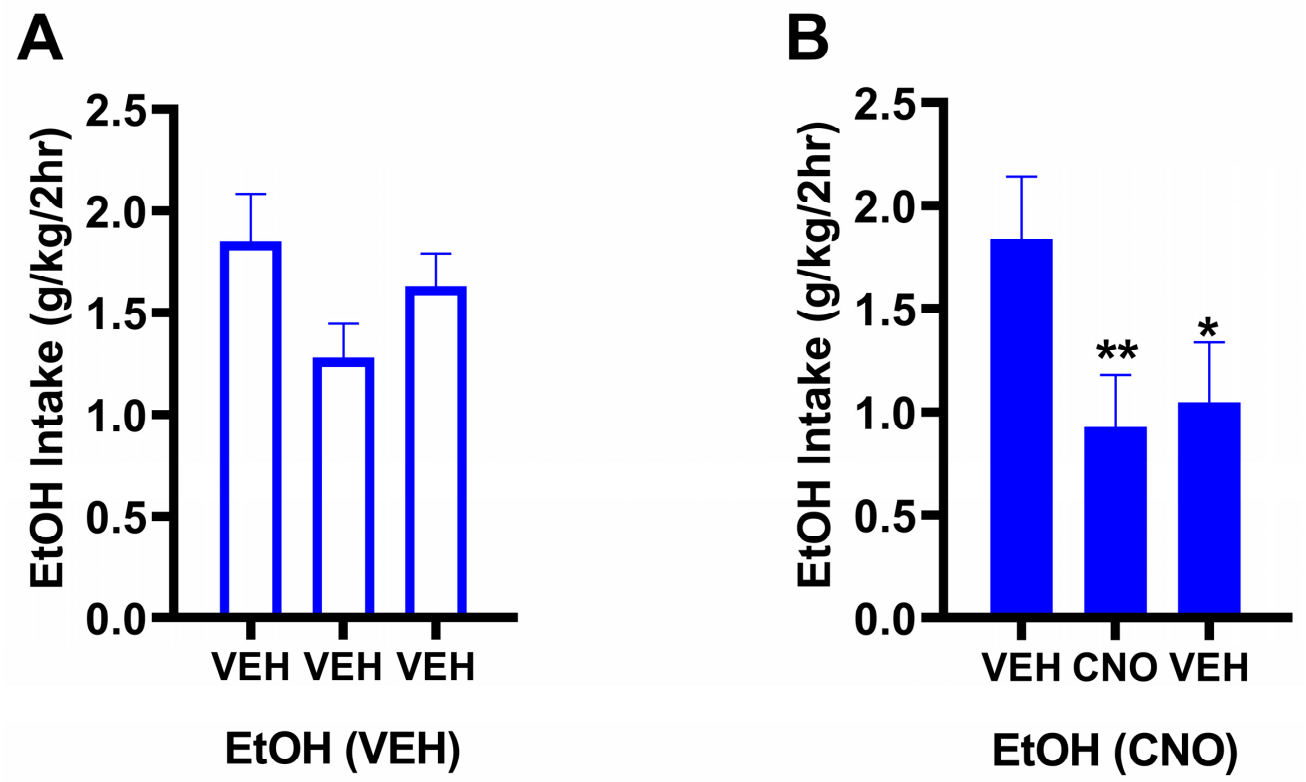

Figure 2. Chronically increasing Gq signaling in the NAc produces lasting reductions in binge-like drinking. Mean ( \pm SEM) 2-h intake during the Drinking in the Dark (DID) paradigm is shown as a function of the three treatment periods. The first bar is data from week 1 to measure baseline intake in response to vehicle, the second bar is data from weeks $2-5$ to measure intake in response to chronic $\mathrm{VEH}(\mathbf{A})$ or $\mathrm{CNO}(\mathbf{B})$, and the third bar is data from week 6 to measure intake in response to VEH again (and determine whether there were lasting effects of CNO). (A) No significant decreases in binge-like $\mathrm{EtOH}$ intake were observed in response to chronic treatment with vehicle throughout the duration of the experiment. (B) Robust, significant reductions in binge-like $\mathrm{EtOH}$ intake were observed in response to $\mathrm{CNO}$ treatment and these reductions persisted during the washout period (one way ANOVA-F $(2,35)=10.49, p<0.01$; post-hoc test revealed significant differences for VEH baseline vs. CNO ${ }^{* *} p<$ $0.01)$ and VEH baseline vs. VEH washout $\left.\left({ }^{*} p<0.05\right)\right)$.

We also performed Sholl analyses for these same neurons to quantify neuronal dendritic complexity. We analyzed intersections (Figure 3I,J,K), length (Figure 3L-N), nodes (Figure 3O-Q) and ends (Figure $3 \mathrm{R}-\mathrm{T})$ by radius $(10 \mu \mathrm{m})$, and examined each radius individually with multilevel analyses and corrections for multiple comparisons. Since the primary effect seen in the neuron summary was an effect of EtOH in the vehicle injection condition, this is the primary comparison analyzed. We found that there were sporadic significant differences in radii, with no overall pattern of effects in any variable (statistics for the analyses are listed in Supplementary Table S5). Therefore, we elected to also examine the area under the curve (AUC). In this analysis, we found a similar effect as we did with the morphometric parameter analysis, as there was a greater AUC in the EtOH exposed groups when compared with $\mathrm{H}_{2} \mathrm{O}$ exposed groups, an effect which was significant in the nodes (Figure 3Q) and ends (Figure 3T), and approached significance in intersections (Figure 3K) and length (Figure 3N). 
Also similar to the morphometric parameter analysis was that this difference between EtOH and $\mathrm{H}_{2} \mathrm{O}$ exposure was no longer seen in the $\mathrm{CNO}$ injection condition, although there was no interaction between the injection and exposure, or effect of the injection.
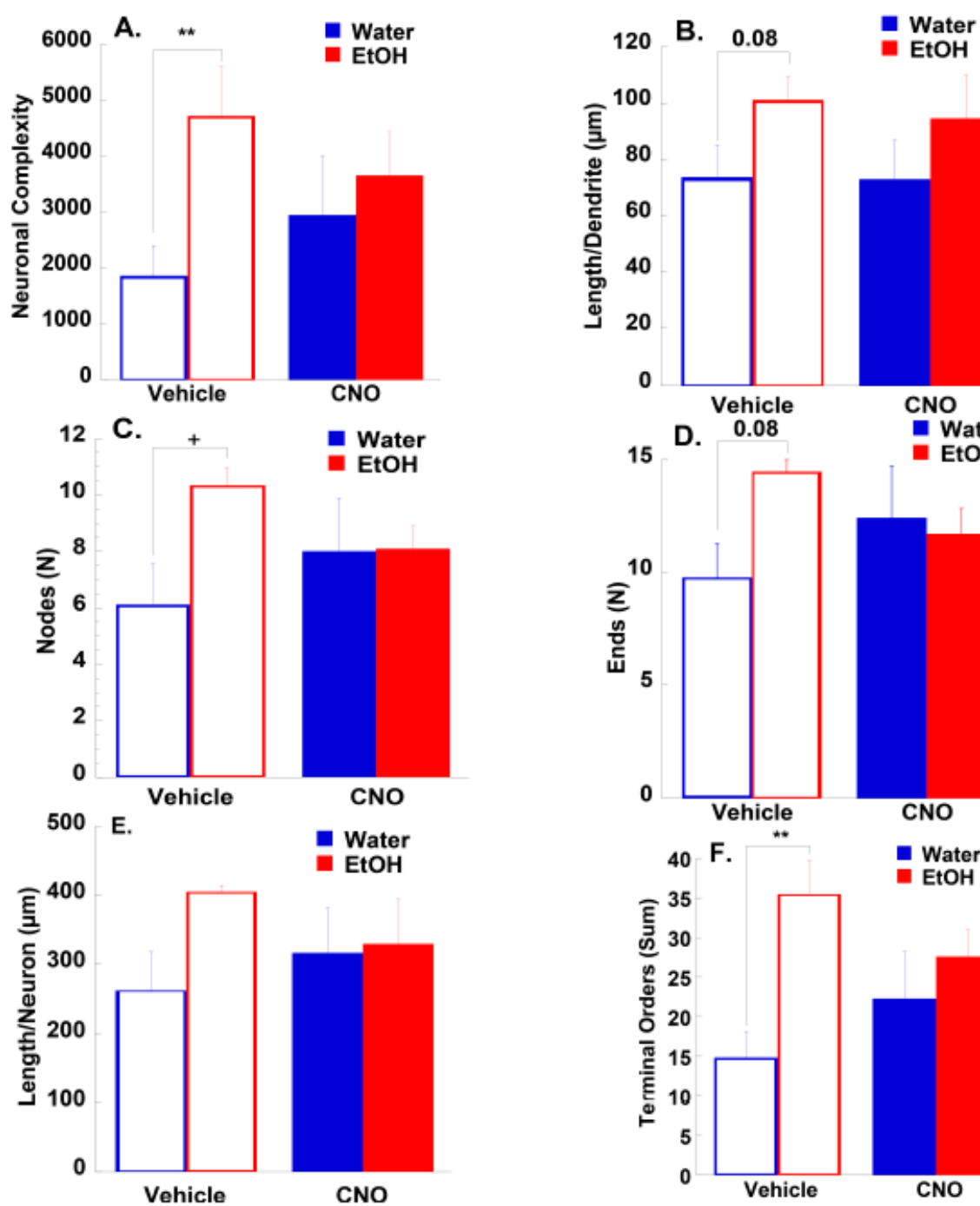

15

D. 0.08
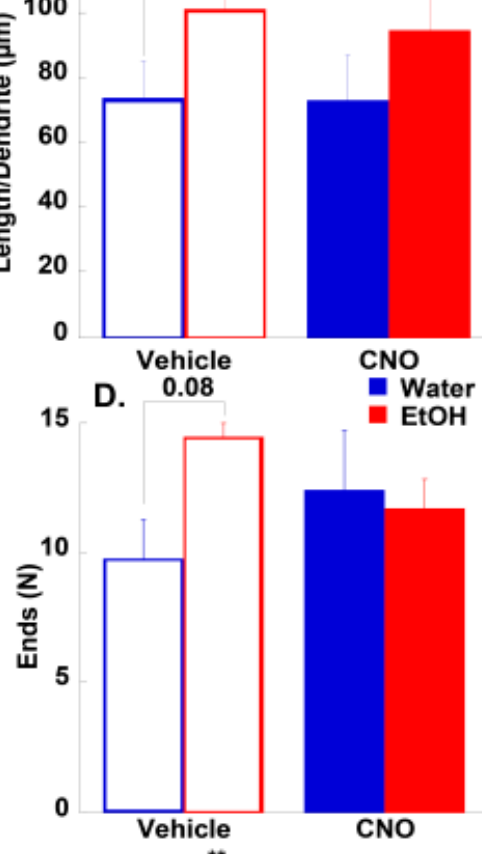

Water

EtOH
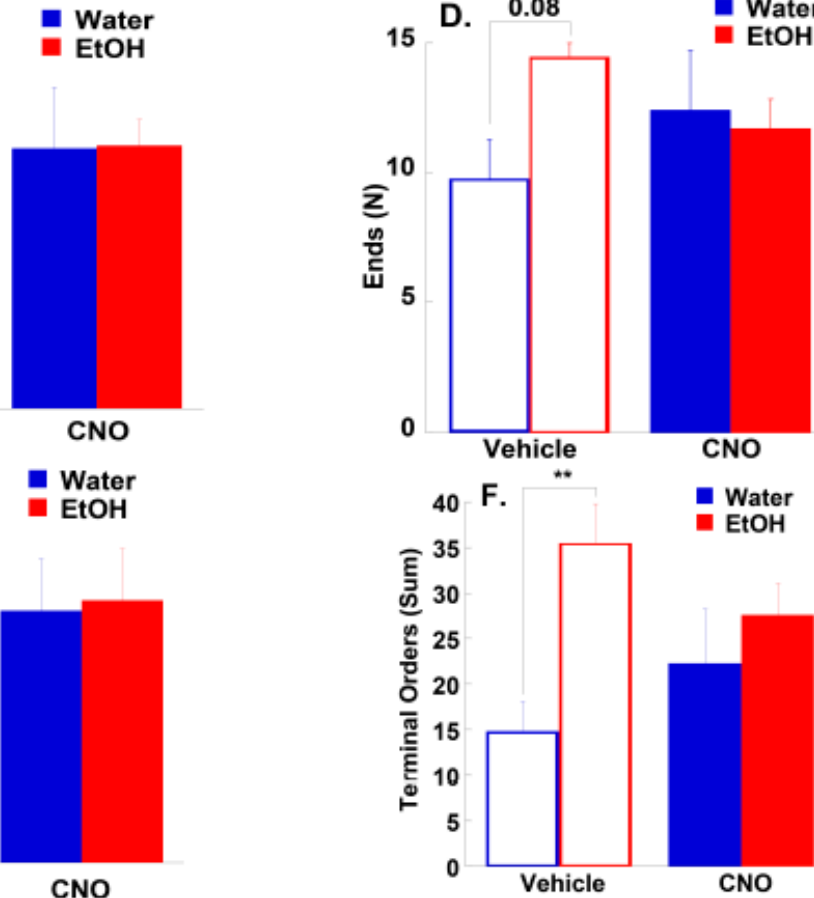

Water

EtOH

5 G.

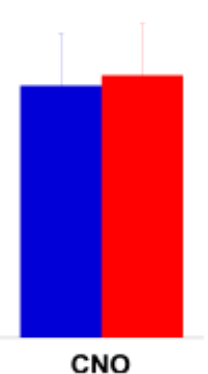

$60 \mathrm{H}$.

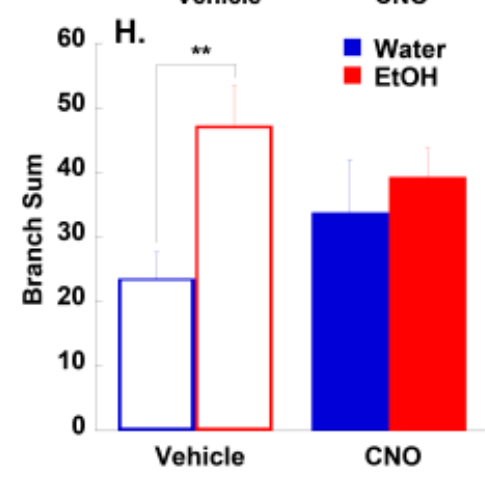

Figure 3. Cont. 


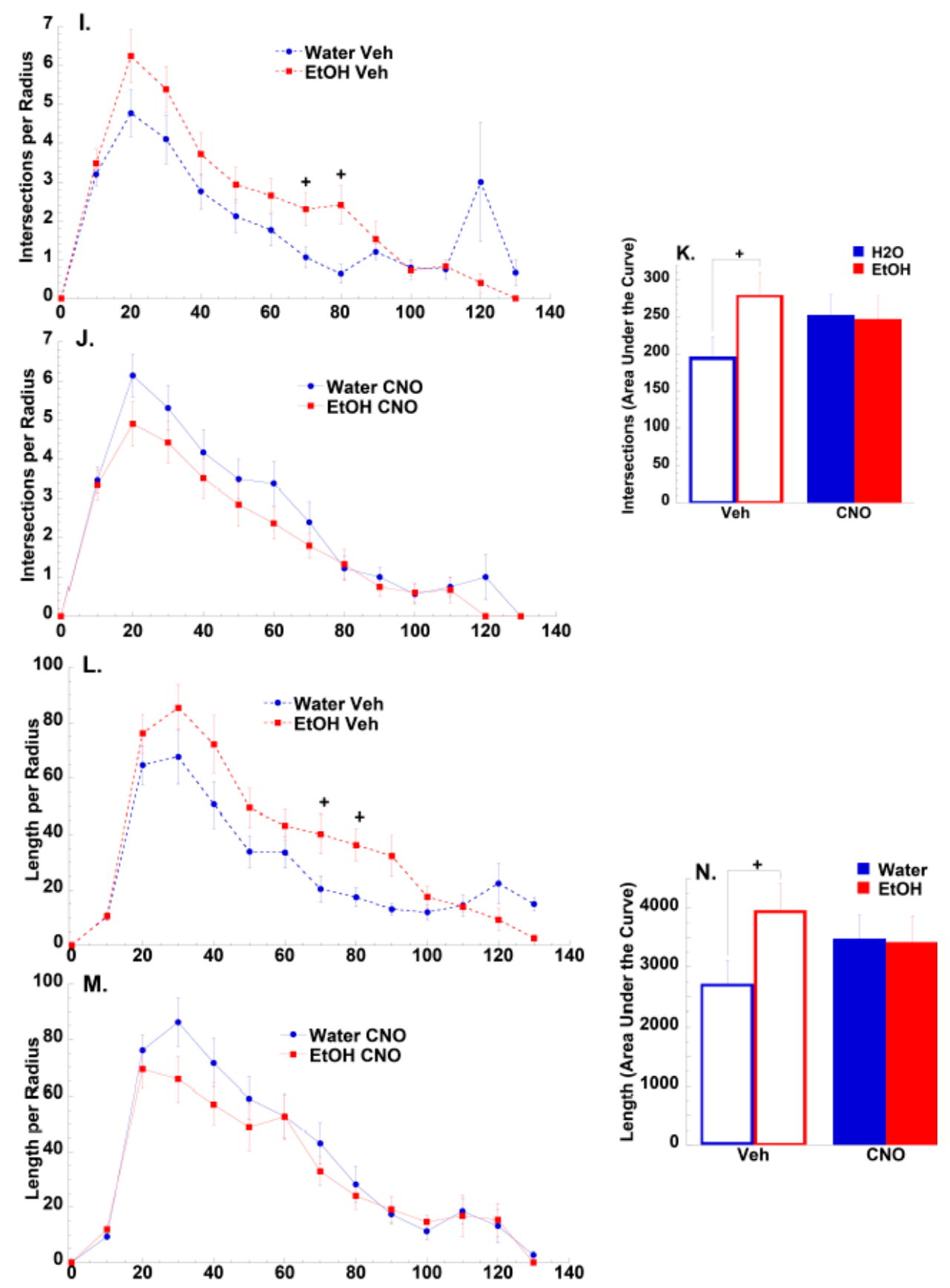

Figure 3. Cont. 


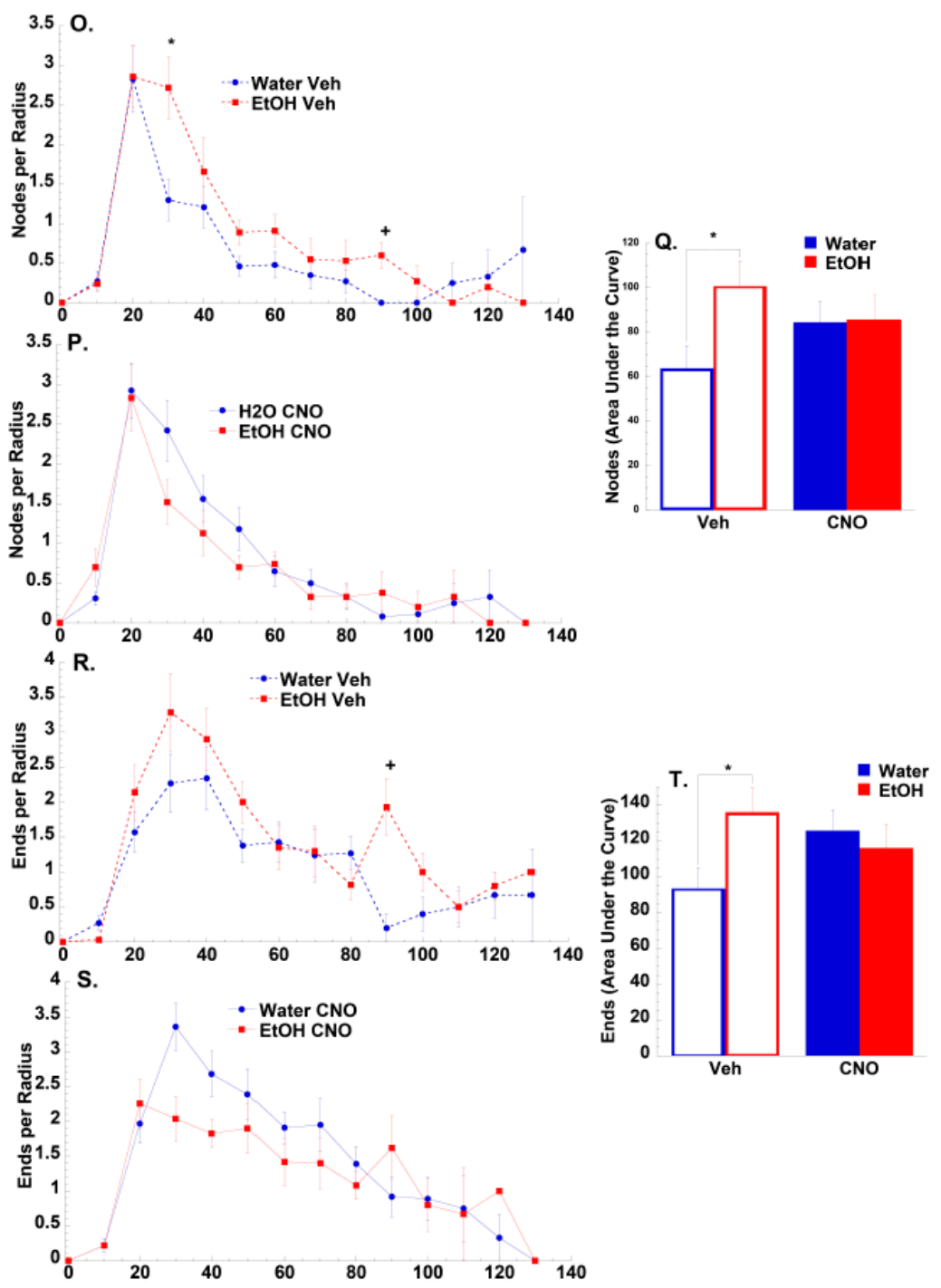

Figure 3. Effects of chronic Gq Designer Receptors Exclusively Activated by Designer Drugs (DREADD) stimulation and drinking on neuronal morphology. Morphometric parameters analyzed for the dendrites of medium spiny neurons. Morphometric parameters of dendrites of nucleus accumbens (NAc) medium spiny neurons of Adult High Drinking in the Dark line 1 (HDID-1) mice injected with either $\mathrm{CNO}$ or a vehicle, and exposed to either $\mathrm{EtOH}$ or $\mathrm{H}_{2} \mathrm{O}$ were analyzed via Neurolucida Explorer. (A) Complexity, calculated via the equation (sum of terminal orders + number of terminals) * (total dendrite length/number of dendrites). (B) Average dendrite length (total length/dendrite quantity) in $\mu \mathrm{m}$. (C) Number of nodes per neuron. (D) Number of ends per neuron. (E) Total dendrite length per neuron, in $\mu \mathrm{m}$. (F) Sum of the terminal orders (the number of sister branches encountered when tracing a dendrite from the tip back to the cell body). (G) Number of dendrites per neuron. (H) Branch sum per neuron (the total number of segments between nodes, a measure of cell branching). (I-T) shows Sholl analysis and area under the curve (AUC) of dendrites of nucleus accumbens medium spiny neurons from HDID-1 mice injected with either CNO or a vehicle, and exposed to either $\mathrm{EtOH}$ or $\mathrm{H}_{2} \mathrm{O}$, which were analyzed with Neurolucida Explorer. Radii were $10 \mu \mathrm{m}$ each and the $x$-axis is "Distance from soma in $\mathrm{uM}^{\prime \prime}$. Intersections per radius of $\mathrm{H}_{2} \mathrm{O}$ Veh vs. EtOH Veh (A), $\mathrm{H}_{2} \mathrm{O}$ Veh vs. EtOH CNO (B), and AUC (C). Length per radius of $\mathrm{H}_{2} \mathrm{O}$ Veh vs. EtOH Veh (D), $\mathrm{H}_{2} \mathrm{O}$ Veh vs. EtOH CNO (E), and AUC (F). Nodes per radius of $\mathrm{H}_{2} \mathrm{O}$ Veh vs. EtOH Veh $(\mathbf{G}), \mathrm{H}_{2} \mathrm{O}$ CNO vs. EtOH CNO (H) and AUC (I). Ends per radius of $\mathrm{H}_{2} \mathrm{O}$ Veh vs. EtOH Veh (J), $\mathrm{H}_{2} \mathrm{O}$ CNO vs. EtOH CNO (K) and AUC (L). Shown in each graph is the mean \pm SEM (for neuron number per group see Supplementary Materials). Multilevel analyses were followed with multiple comparison corrections using the Benjamini-Hochberg approach to adjust $p$-values to the false discovery rate (FDR) (see Table 1 ). ${ }^{* *} q$-value $<0.01,{ }^{*} q$-value $<$ 0.05 (after FDR corrections),$+=p$-value $<0.05$ (before FDR corrections). All statistics are in Table S5. 


\subsubsection{Transcriptomic Results}

To identify gene expression changes related to binge drinking and CNO treatment, differential expression analysis was performed using four groups from experiment 2 . These four groups were defined as untreated binge-drinking $(\mathrm{EtOH}(\mathrm{VEH}))$, treated binge-drinking $\mathrm{EtOH}(\mathrm{CNO})$, treated water drinking $\left(\mathrm{H}_{2} \mathrm{O}(\mathrm{CNO})\right)$ and control water drinking $\left(\mathrm{H}_{2} \mathrm{O}(\mathrm{VEH})\right)$ animals. Comparisons made between groups involved the pairwise comparison of all groups with the control $\mathrm{H}_{2} \mathrm{O}(\mathrm{VEH})$ only. In this way, we identified differentially expressed genes (DEGs) from all groups relative to the control (Figure S1, Table S1). The lists of DEGs were then compared for either overlapping or unique genes based on each group (Tables S1 and S2). The overlap in these DEGs is illustrated in Figure 4A. Here, 688 (out of 1473) significant DEGs were uniquely differentially expressed in EtOH (VEH). In EtOH (CNO) 1431 (out of 2377) genes were uniquely differentially expressed and in $\mathrm{H}_{2} \mathrm{O}$ (CNO) 612 (out of the 1157) genes were differentially expressed. The overlap for DEGs was highest between the $\mathrm{EtOH}(\mathrm{VEH})$ and $\mathrm{EtOH}(\mathrm{CNO})(n=513)$ and lowest between $\mathrm{EtOH}(\mathrm{VEH})$ and $\mathrm{H}_{2} \mathrm{O}(\mathrm{CNO})$ $(n=112)$. Expression patterns for the unique DEGs identified for $\mathrm{EtOH}(\mathrm{VEH}), \mathrm{EtOH}(\mathrm{CNO})$ and $\mathrm{H}_{2} \mathrm{O}$ (CNO) are shown in Figure 4B. Each row represents a group, while each column represents a gene transcript. Transcripts are color-coded based on log-2 fold change from positive (blue) to negative (red). Gray indicates minimal change in expression. To identify genes that were significantly changed with chronic binge drinking and ameliorated by $\mathrm{CNO}$, we calculated the pairwise Euclidian distance in expression patterns (for the 688 unique DEGs identified for the EtOH (VEH) group) and present the data in Figure S2 and Table S2 and discuss these findings in the Supplementary Materials.

To identify the transcriptomic mechanisms of morphological changes observed in previous analyses, we identified gene expression changes of key molecular mediators of structural plasticity (details and data tables are provided in the Supplementary Materials and methods). The heatmap in Figure 4C illustrates the expression patterns of 57 genes previously associated with increased (or decreased) neuronal structure. Columns indicate the comparisons performed for $\mathrm{EtOH}$ (VEH) vs. $\mathrm{H}_{2} \mathrm{O}$ (VEH) or EtOH (CNO) vs. $\mathrm{H}_{2} \mathrm{O}(\mathrm{CNO})$. DEG lists and expression values are listed in Supplementary Table S5. The significance of each gene relative to its comparison within the column is indicated, as ${ }^{*} p<0.05,{ }^{* *} p<0.01$. Here, 11 DEGs were identified between the two comparisons: 8 were found to be differentially expressed in $\mathrm{EtOH}(\mathrm{VEH})$ vs. $\mathrm{H}_{2} \mathrm{O}(\mathrm{VEH})$ and 3 in $\mathrm{EtOH}(\mathrm{CNO})$ vs. $\mathrm{H}_{2} \mathrm{O}(\mathrm{CNO})$. No overlap was observed within the DEGs between the two comparisons. Of the DEGs identified, all were associated with increased structural changes, excluding Dlg4 and Mef2d. The 8 DEGs identified within the $\mathrm{EtOH}(\mathrm{VEH})$ vs. $\mathrm{H}_{2} \mathrm{O}(\mathrm{VEH})$ comparison included Nrtk2, Wasf2, Dlg4, Grin2b, Grin2a, Was, Actl6a and Mir132, where Nrtk2, Wasf2, Grin2b, Grin2a, and Was were significantly upregulated and Dlg4, Actl6a and Mir132 were downregulated. The 3 DEGs identified within the EtOH (CNO) vs. $\mathrm{H}_{2} \mathrm{O}(\mathrm{CNO})$ comparison included Mef2d, Camk2g and Pak1, where Camk $2 g$ and Pak1 were significantly upregulated and $M e f 2 d$ was downregulated. In conjunction with the morphological analyses presented, these results suggest that expression of the mediators of structural plasticity are altered with ethanol drinking and ameliorated with $\mathrm{CNO}$ treatment.

The results of gene ontology (GO) term enrichment for biological processes in ETOH (VEH) are highlighted in Figure 5A. GO annotation of the ranked DE genes in EtOH (VEH) revealed a significant enrichment in gene-associated processes such as macromolecule metabolism, protein metabolism, cellular macromolecule metabolism, positive regulation of cellular processes, nitrogen compound metabolism, regulation of macromolecule metabolism and cellular protein metabolism among others (Supplementary Table S3). Genes overrepresented in these categories are shown in the clustergram in Figure 5A, where enriched terms are rows and input genes are columns. Cell colors in the matrix indicate which gene is ranked first within each associated GO term. The color depth of the cell corresponds to the legend and indicates the p-value of the enrichment term. Dark red indicates high significance and light red indicates low. The top 9 genes ranked first in the top 20 significant GO terms were Cpsf3l, Hdac4, Fgfr3, Xpo4, Emilin2, Kiss1r, Trak2, Camta1 and Fam135b. In particular, Hdac4 was ranked first in 13 of the top 20 GO categories. Among all categories, histone deacetylase 4 (Hdac4), 
a class II HDAC that deacetylates lysine residues of core histones and represses transcription via MEF2C and MEF2D, tended to be most prevalent in those related to the regulation of metabolic cellular processes. Comparison of the total normalized gene expression of Hdac4 between groups is presented in Figure 5B. We observed a significant difference in expression between $\mathrm{H}_{2} \mathrm{O}$ and EtOH groups receiving vehicle treatment (two-way ANOVA: Fluid $\times$ Treatment interaction- $\mathrm{F}(1,41)=4.45, p<0.05$; main effect of fluid $-\mathrm{F}(1,41)=6.23, p<0.05$; post-hoc test revealed $\mathrm{EtOH}(\mathrm{VEH})$ vs $\left.\mathrm{H}_{2} \mathrm{O}(\mathrm{VEH}), p<0.05\right)$.

\section{A}
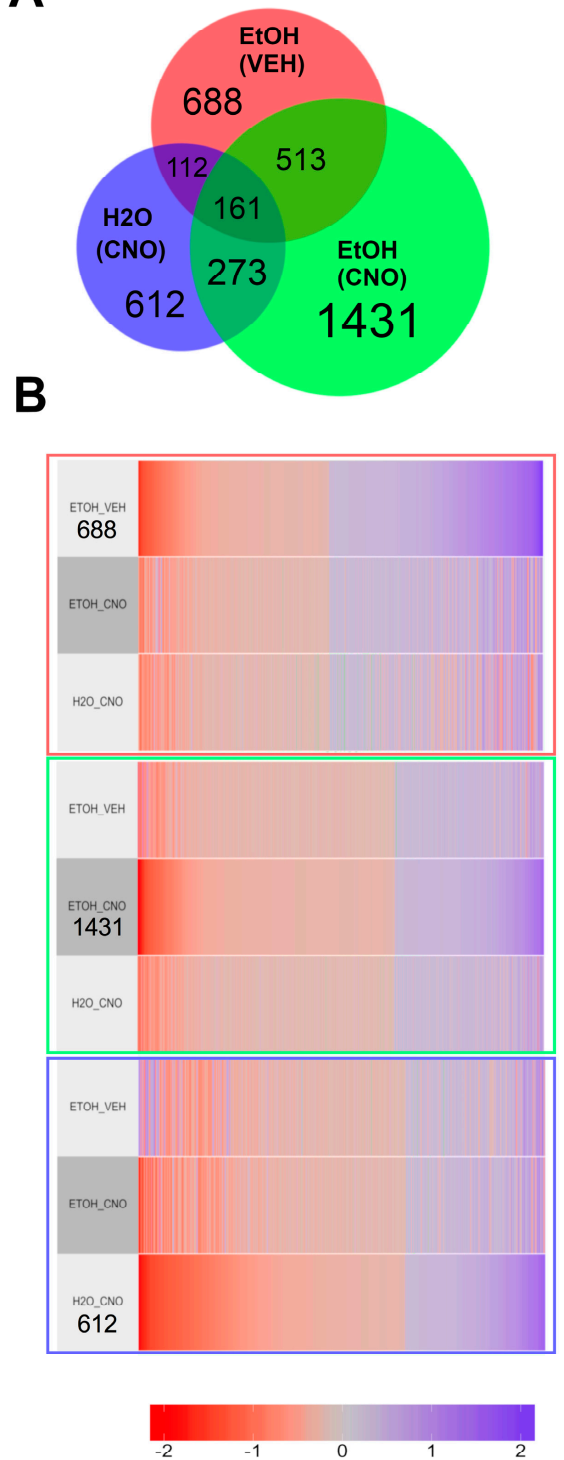

\section{C}
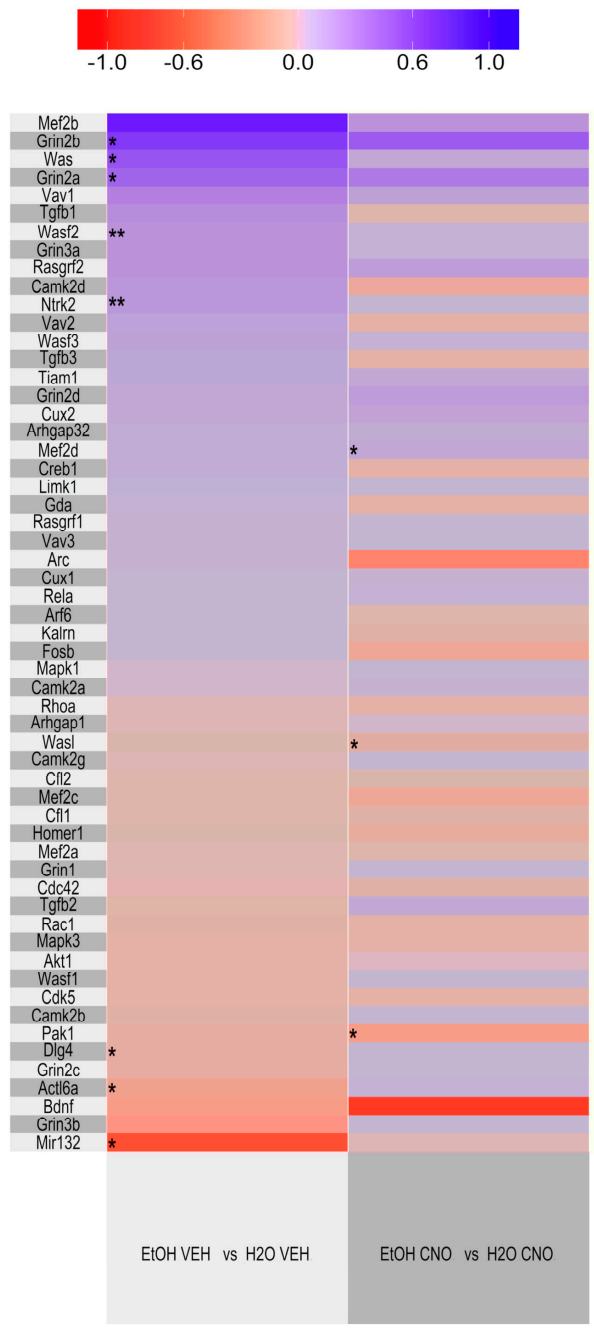

Figure 4. Gene expression changes produced by chronic binge drinking and stimulation of Gq signaling in the NAc. (A) The Venn diagram represents overlapping or unique differentially expressed genes (DEGs) $(p<0.05)$ in each group based on a pairwise comparison of all groups to $\mathrm{H}_{2} \mathrm{O}(\mathrm{VEH})$ mice. (B) Patterns of DEGs associated with binge-like EtOH drinking identified in (A) across groups are visualized by heatmaps, where blue $=$ upregulation, red $=$ downregulation, and gray $=$ minimal to no change in expression. The top panel of (B) shows the 688 DEGs associated with EtOH (VEH), the middle panel shows the 1431 associated with $\mathrm{EtOH}(\mathrm{CNO})$ and the bottom panel shows the 612 associated with $\mathrm{H}_{2} \mathrm{O}(\mathrm{CNO})$. (C) To identify the molecular mechanisms mediating changes in neuronal morphology induced by chronic binge drinking that were ameliorated with $\mathrm{CNO}$, we have highlighted the expression patterns of 57 key genes known to mediate neuronal morphology. DEGs are shown as * $p<0.05,{ }^{* *} p<0.01$ and the comparisons are labeled within the columns. 
A

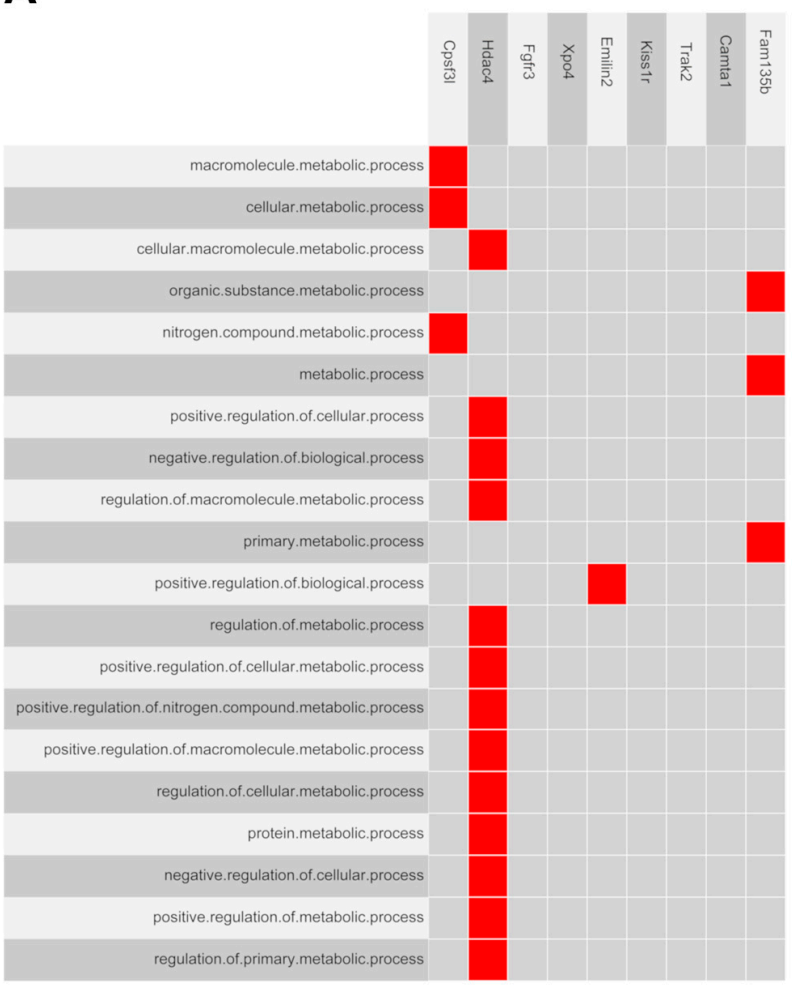

B

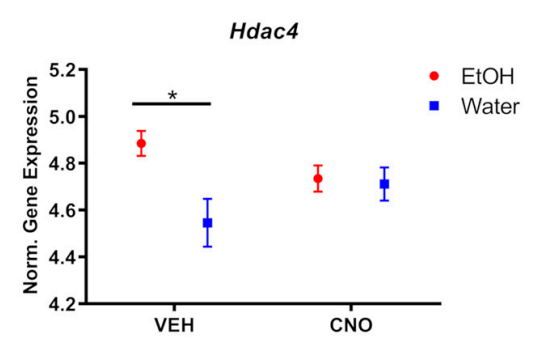

C

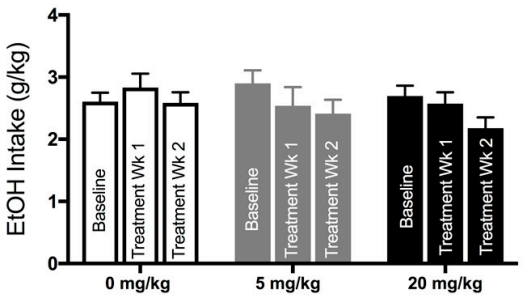

D

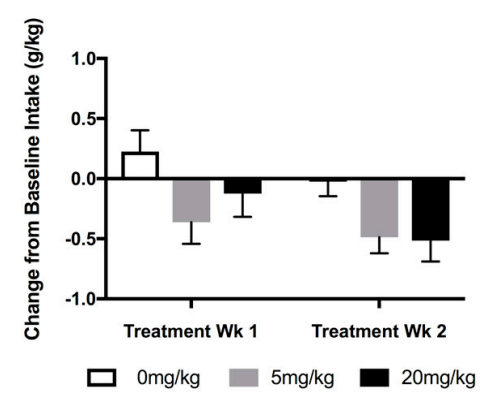

Figure 5. Differential expression of Hdac4 and treatment with HDAC4/5 inhibitor, LMK-235, reduced binge-like alcohol drinking. (A) A clustergram is shown to visualize results from GO performed on ranked DEGs identified from $\mathrm{EtOH}(\mathrm{VEH})$. Enriched terms are listed in rows and the corresponding genes ranked first in each term are shown in each column. Red indicates a gene is ranked first within a term. Grey indicates a gene is not ranked first. (B) Limma normalized gene expression levels of Hdac4 are shown for each treatment and fluid group. A significant increase in normalized expression was observed between $\mathrm{H}_{2} \mathrm{O}$ and $\mathrm{EtOH}$ groups receiving vehicle treatment $\left({ }^{*} p<0.05\right)$. (C) Average weekly ethanol intake after treatment with 0,5 or $20 \mathrm{mg} / \mathrm{kg}$ LMK-235. Two-way ANOVA revealed a significant main effect of treatment period on ethanol intake $(\mathrm{F}(2,152)=5.16, p<0.01)$. (D) Ethanol intake presented as the average difference score from baseline intake for each treatment week. Here, significant reductions were observed for both doses over a two-week period (Two-way ANOVA (dose $\times$ treatment week) revealed a significant main effect of dose and a significant main effect of treatment week (dose: $\mathrm{F}(2,76)=3.60, p<0.05$; treatment week: $\mathrm{F}(1,76)=2.44, p<0.05)$ ).

WGCNA was performed to identify the impact of chronic binge-like drinking and the lasting effects of CNO treatment on coordinated regulated gene expression networks. Though not discussed here, the results implicated the direct susceptibility of gene expression in a specific module to binge-like drinking and highlighted it as a point of interest (results in Supplementary Materials, Table S4 and Figure S3).

\subsection{Experiment 3: Pharmacologically Testing the Role of the Overrepresented Gene, Hdac4, in Binge-Like Drinking}

The effects of HDAC4/5 inhibitor, LMK-235, on binge-like alcohol drinking were significant. Notably, reductions in binge drinking became more apparent over time, suggesting transcriptional mechanisms. When comparing ethanol intake across baseline and treatment weeks, a two-way ANOVA revealed a significant main effect of treatment period on ethanol intake (Figure $5 \mathrm{C} ; \mathrm{F}(2,152)=5.16, p<$ 0.01). Previous work has shown that the HDAC4/5 inhibitor, LMK-235, requires several administrations 
to exert its effects on signaling and morphology. We followed up on this finding by calculating a change score for each animal (by subtracting the average intake during treatment week 1 or week 2 from the average intake during baseline; shown in Figure 5D). Two-way ANOVA (dose $\times$ treatment week) revealed a significant main effect of dose, as well as a significant main effect of treatment week (dose: $\mathrm{F}(2,76)=3.60, p<0.05$; treatment week: $\mathrm{F}(1,76)=2.44, p<0.05)$.

\section{Discussion}

Despite the prevalence of AUD and associated binge-related alcohol abuse, individuals often receive limited or no treatment. Currently, three FDA-approved medications are available for AUD treatment: disulfiram, acamprosate and naltrexone [48]. However, these medications are often underutilized, only improve symptoms in a small fraction of treated individuals, and any improvements do not last beyond treatment [49]. Further, although DBS of the NAc appears to be a potentially promising therapeutic approach, it is quite invasive and does not produce lasting reductions in drinking or craving. Thus, it is clear there exists a need for therapeutic approaches that produce robust and lasting effects. Here, we have shown that chronic binge-drinking induces lasting changes in neuronal morphology and gene expression networks, which can be ameliorated via chronic stimulation of Gq signaling (via CNO/hM3Dq) in the NAc. Furthermore, we found that we could pharmacologically target HDAC4, a gene that is altered with chronic binge-like drinking and ameliorated with $\mathrm{CNO} / \mathrm{hM} 3 \mathrm{Dq}$, to reduce binge drinking.

\subsection{Animal Models of Binge Drinking and DREADDs}

We and others have previously used HDID-1 mice as a reliable and predictive genetic model for high intensity binge-like drinking. This is due to their ability to achieve pharmacologically relevant blood alcohol levels (BALs), exhibit behavioral impairment after binge-like drinking, and exhibit relapse and withdrawal behavior following binge-like drinking sessions [6]. Genetic selection for high BALs has not altered the preference of mice to other tastants, nor has it affected their ability to metabolize alcohol [3]. Herein, the DID paradigm was employed for its high-throughput, limited access approach which did not require the use of tastants to motivate excessive levels of ethanol consumption during the dark cycle. In our study, we observed robust reductions in ethanol intake of female HDID-1 mice expressing hM3Dq in the NAc when treated with CNO during a DID limited access paradigm. These effects were observed for the entirety of the CNO treatment period (28 days), suggesting mice expressing hM3Dq in the NAc did not develop tolerance or show sensitization to the effects of chronic $\mathrm{CNO}$ administration. Moreover, the decreased intake persisted for at least 7 days during the vehicle "washout" period. We did not observe any change in ethanol drinking for female mice expressing GFP or hM4Di in the NAc, suggesting our findings are specific to HDID-1 mice expressing hM3Dq. However, there may exist sex differences, or paradigm-specific effects, in the effects of CNO/DREADDs for the NAc. Cassataro et al. (2014) found that CNO reduced binge-like drinking in C57BL/6J males expressing hM4Di in the NAc [50]. Direct comparison of these studies should be cautioned due to differences in methodology (length of ethanol access was $2 \mathrm{~h}, \mathrm{CNO}$ was administered IP, and only females were used in the current DREADD study, and length of ethanol access was 2 and $4 \mathrm{~h}, \mathrm{CNO}$ was administered via tap water to male mice in [50]. We are currently following up on these differences by conducting studies with both males and females to evaluate the role of sex as a biological factor. Taken together, these results provide supportive evidence for chronic Gq stimulation of the NAc as a causal factor in molecular and behavioral plasticity associated with lasting reductions of binge-like drinking in female mice.

\subsection{Effects of Binge-Drinking and DREADDs on NAc Neuronal Morphology}

We found that chronic binge-like drinking resulted in increased complexity of medium spiny neurons in the NAc of HDID-1 female mice, an effect that was ameliorated by chronic treatment with CNO. Interestingly and unexpectedly, no effect of CNO was observed in water drinking mice, 
suggesting that chronically increasing Gq signaling in the NAc does not produce robust and lasting changes in neuronal complexity. We were unable to identify any published reports on the effects of DID or binge-like drinking on neuronal morphology in the NAc (or any other brain region) for comparison. However, there are several published studies evaluating the effects of chronic intermittent ethanol vapor on neuronal morphology in different brain regions. Most relevant to this work are studies by Wang et al. (2015), Uys et al. (2016), and DePoy et al. (2013) evaluating the effects of chronic intermittent ethanol vapor (CIE) on aspects of neuronal morphology in the dorsal and ventral striatum [42,51,52]. In brief, these studies report that CIE results in increased spine density, increased dendritic diameter, increased dendritic length and number of processes. Moreover, alcohol-induced increases in neuronal complexity in the dorsal striatum are correlated with increased AMPA receptor activity specifically in the dopamine D1 receptor expressing MSNs (medium spiny neurons), suggesting that alcohol enhances the activity of D1 MSNs in the direct pathway [52]. Notably, striatal circuits associated with D1- and D2-expressing MSNs are not equivalent for the dorsal and ventral striatum [53]. The role of D1- and D2-expressing MSNs of the NAc core in binge-like drinking is currently under study in our laboratory. In summary, although binge-like drinking in DID is likely modeling different aspects of AUD than CIE, it appears that both alcohol drinking models result in morphological changes in the dorsal and ventral striatum that manifest as increased neuronal complexity. Although additional studies are needed to better understand the effects of alcohol on morphology in different species, strains, sexes, and drinking paradigms.

Neuroadaptative changes, such as increased complexity, in NAc MSNs induced by ethanol exposure are the products of dysregulation in signaling systems, gene transcription and protein expression at the cellular level [54]. Previously, it has been shown that both opiate and stimulant drugs of abuse disrupt numerous genes that encode cytoskeleton regulatory proteins as well as their respective transcriptional factors [40]. These include cell surface receptors, adhesion molecules, signaling molecules, scaffolding proteins, GTPases regulating the actin cytoskeleton and guanine nucleotide exchange factors (GEFs) activated by GTPases. These mediators are associated with directional control of neuron morphogenesis. For example, neurotrophic factor components such as CREB and MAPK have been shown to increase dendrite branching, while scaffolding proteins such as PSD95 have been shown to act as stop signals for proximal dendritic branching [41]. In our work, we observed a significant upregulation of genes associated with increased dendritic branching and complexity such as Ntrk2 (encodes BDNF tyrosine receptor kinase-B (TRKB)), Wasf2, Was (genes encoding Wiskott-Aldrich Syndrome proteins), Grin $2 a$, and Grin $2 b$ (genes encoding NMDA receptors) with chronic binge drinking (as compared to water controls). Within the same comparison, we also observed a significant downregulation of genes Dlg4 (encodes the post synaptic density scaffolding protein, PSD95), Mir132 (CREB mediated microRNA) and Actl6a (encodes the actin-related protein-4 ARP4). These significant changes in expression patterns were absent within the $\mathrm{EtOH}(\mathrm{CNO})$ vs. $\mathrm{H}_{2} \mathrm{O}$ $(\mathrm{CNO})$ comparison. In the context of untreated binge drinking, these changes in gene expression likely represent the underlying mechanisms for the increased neuronal complexity observed with chronic binge drinking. Further, the absence of these expression patterns with CNO treatment supports that the expression of these genes is ameliorated with CNO. Support for these mechanisms have also been shown by Uys et al., 2016, where their group observed significant changes in NAc core MSN morphology and expression of PSD95 (Dlg4) with chronic intermittent ethanol exposure [42]. Additionally, PSD95 has also been shown to be downregulated by other drugs of abuse such as morphine and cocaine [55-57].

\subsection{Effects of Chronic Binge-Drinking and DREADDs on the NAc Transcriptome}

Reductions in ethanol consumption observed during the washout period of the DID paradigm suggest neuroplastic changes as a result of chronic neuronal stimulation in mice expressing hM3Dq. Transcriptomic analyses were carried out to identify differentially expressed genes (DEGs) for each group (Figure S1) and used to identify genes associated with binge-like drinking that were ameliorated 
by CNO (Figure S2). Using DEG analysis, genes with significantly altered levels of expression were identified and contextualized within biological networks using Gene ontology analysis (GO). Weighted gene covariance network analysis (WGCNA) was used to construct a scale-free network and identify modules. Genes with a high degree of network connectivity (hubs) that correlated with binge-like drinking were used to identify candidate therapeutic targets (Figures S3 and S4; additional results and discussion in Supplementary Materials). Gene overlap identified from DE implicated changes in gene expression that were either treatment and/or fluid type specific. The 688 genes changed solely in the group EtOH (VEH) indicated genes whose expression was perturbed exclusively by chronic binge-like alcohol drinking. This is the first time that NAc transcriptomic changes related exclusively to either chronic binge drinking or chronic CNO stimulation of hM3Dq have been identified. For $\mathrm{EtOH}(\mathrm{CNO}), 1431$ genes were found to be related exclusively to those genes ameliorated by CNO treatment. Further, 612 genes were identified in the $\mathrm{H}_{2} \mathrm{O}(\mathrm{CNO})$ group and represented genes that were related exclusively to chronic CNO-induced activation of hM3Dq.

Progression from acute to chronic alcohol intake is thought to incite changes in gene expression levels that occur to maintain system homeostasis [58]. Chronic exposure to alcohol causes dysregulation of these homeostatic mechanisms leading to alcohol abuse and dependence. Several groups have reported on the effects of selection for alcohol behaviors or alcohol drinking on the transcriptome (for various brain regions and strains/species). Mulligan et al. (2011) examined gene expression after binge drinking (immediately after a 4-day DID), and detected a strong relationship between the BEC and a striatal network of gene modules enriched in gene annotations for protein binding and oxidative phosphorylation. Hitzemann et al. (2017) reported a significant overlap between striatal gene expression associated with individual variation (measured 3 weeks after a 4-day DID) and risk for excessive drinking (genes associated with selection as measured in naïve mice) [10]. Many of these genes were related to NMDA-mediated synaptic plasticity. Furthermore, Ferguson et al. (2019) found many changes in brain gene expression between ethanol-naïve HDID-1 and HS/Npt mice, specifically in glutamatergic and GABAergic signaling pathways in the NAc (shell) [59]. The brain regions used in Ferguson et al. (2019) represent circuitry well known to be involved in motivated behaviors, as well as alcohol drinking [59]. Hdac4 was identified in selection responsive modules as functionally overrepresented. We identified Hdac4 as a gene of interest in our study and have discussed this below.

We observed 688 genes uniquely differentially expressed in the EtOH (VEH) group, where those exhibiting the most extreme changes in expression were ameliorated in $\mathrm{CNO}$ treatment groups. GO analysis of DEGs identified for the EtOH (VEH) group identified the importance of Hdac4 expression in binge drinking. HDAC4 is a class IIa histone deacetylase that represses transcription when tethered to a promoter (via a multiprotein complex with MEF2C and MEF2D). In pathways such as cellular macromolecule metabolism, positive regulation of cellular processes, negative regulation of biological processes and regulation of macromolecule metabolism, Hdac4 was consistently found to be overrepresented. Additionally, we observed gene representation related to circadian and neuroimmune pathways, as others have previously reported $[8,60,61]$. Because Hdac4 was present in the majority of significant GO pathways, we hypothesized that Hdac4 played a large role in a number of biological pathways related to chronic binge drinking. We were encouraged to use this approach to test whether pharmacologically targeting HDAC4 could reduce binge-like drinking.

\subsection{Pharmacologically Targeting HDAC4 to Reduce Binge-Like Drinking}

It has been shown that alcohol dependence and withdrawal result in a global reduction of expressed transcripts, accompanied by an increase in expressed transcripts coding for regulators of epigenetic silencing [62]. One such class of enzymes, histone deacetylases (HDAC; transcriptionally silencing enzymes), are inhibited by valproate. Valproate treatment during alcohol withdrawal reduces anxiety in dependent animals [63]. Simon-O'Brien et al. (2012) reported that several classes of HDAC inhibitors and DNA methyltransferase inhibitors decrease alcohol-seeking behaviors [64]. Thus, 
there is growing evidence that epigenetic modifiers and changes in gene expression play a role in alcohol behaviors. Here, we showed that the chronic treatment with the HDAC4/5 inhibitor LMK 235 reduced binge-like drinking in mice selectively bred to drink to intoxication. Based on these results, we inferred that there were changes in transcriptional mechanisms because we observed that reductions in intake developed over several treatment days. Although Hdac4 was selected as a target based on transcriptomic data from female mice, the compound reduced ethanol drinking in both male and female mice. Others have shown that HDAC4 is regulated by neuronal activity and plasticity, and inhibiting HDAC4 via LMK 235 can alter neuronal morphology $[50,65,66]$. Ongoing work is focused on using this data to identify and test compounds with high clinical translational potential.

\section{Summary}

We found chronically increasing NAc activity (4 weeks via CNO/hM3Dq) decreased binge-like drinking. This effect persisted for 7 days post-CNO treatment, suggesting the development of plasticity. We investigated whether this behavioral plasticity could be observed at the morphological and/or transcriptomic levels. We found that chronic binge drinking increased neuronal complexity in the NAc, an effect not present in mice treated with CNO. We detected significant changes in expression of several morphology and plasticity-related genes with binge drinking that were ameliorated with CNO treatment (e.g., Hdac4). We tested whether inhibiting HDAC4 would reduce drinking and found that chronic treatment with LMK235, an HDAC4/5 inhibitor, reduced binge-like drinking. Thus, we were able to target specific molecular pathways using pharmacology to mimic the behavioral effects of DREADDs.

Supplementary Materials: The following are available online at http://www.mdpi.com/2076-3425/10/2/109/s1, Table S1: Differentially expressed gene lists; Table S2: Gene lists for Venn diagram and Heatmaps of gene expression; Table S3: Results of gene ontology analyses; Table S4: Weighted gene covariance network analysis (WGCNA) module information and gene lists; Table S5: Morphology statistics and differentially expressed genes (DEG) related to neuronal morphology; Figure S1: Top genes resulting from DEG analyses. Volcano plots for three group comparisons highlight the top 20 genes with the lowest p-values within each comparison. All comparisons shown, are presented relative to the $\mathrm{H}_{2} \mathrm{O}(\mathrm{VEH})$ control group. (A) DEGs in EtOH(VEH) group, (B) DEGs in $\mathrm{EtOH}(\mathrm{CNO})$ group, and (C) DEGs in $\mathrm{H}_{2} \mathrm{O}(\mathrm{CNO})$ group; Figure S2: Gene expression changes produced by chronic binge drinking and stimulation of Gq signaling in the NAc. To identify DEGs affected by ethanol that were rescued by $\mathrm{CNO}$, an Euclidean distance measure clustering was used. The top 47 genes with greatest variability (pairwise Euclidean distance $>1.5$ standard deviations from mean) in expression patterns unique to EtOH (VEH) are illustrated. Gene lists and expression values are listed in Supplementary Table S2. Here, a majority of these DEGs were significantly enriched in four categories: positive regulation of immune responses, general immune response-regulating signaling pathways, immune response-activating signal transduction and activation of immune responses. This indicated that gene expression changes significantly perturbed by binge-drinking, disproportionately impact expression of genes in neuroimmune pathway regulation; Figure S3: Effects of DREADDs and Drinking on Gene Networks. (A) Gene dendrogram showing the co-expression modules defined by labeled colors. Gene dis-similarity is shown as height. (B) Table describing module characteristics, hub genes and their descriptors, and module membership of unique DEGs identified in Figure 3. (C) Eigengene dendrogram showing the correlation of modules as labeled colors corresponding to (B). The dis-similarity of modules is expressed as height. (D) Matrix with the Module-Trait Relationships with expression values (and corresponding p-values) between the detected modules (rows) and fluid type and treatment traits (columns). Blue Module-Trait relationships indicate a strong upregulation, while red indicates a strong downregulation (and white indicates little or no change). ${ }^{*} p<0.05,{ }^{* *} p<0.01$.

Author Contributions: A.R.O. designed the studies, A.R.O., D.Y.P., S.P.F., C.M.G., K.G.T., E.J.F., and M.G. conducted the studies and analyses, A.R.O. and D.Y.P. wrote the manuscript. All authors have read and agreed to the published version of the manuscript.

Acknowledgments: This paper gratefully acknowledges the help and support provided by the following people: Ovidiu (Dan) Iancu, John Crabbe, Rachel Arey, Robert Hitzemann, Robert Searles, and Miranda Lim. The authors gratefully acknowledge their research funding. To ARO: U.S. Department of Veterans Affairs Awards IK2BX002488, NIH - AA013519, AA10760, and AA026997, Andrews Genomics Fund, NARSAD Young Investigator Award, Collins Medical Trust. To DP and KT: Build EXITO program Grant 5TL4GM118965; To DP: Ronald E. McNair Scholar Program. To SPF: NIH AA020926 and AA024836. To CG: AA024950. To MG: NIH - AA021468 and AA022948, VA Merit Review Award I01BX001819.

Conflicts of Interest: The authors declare no conflicts of interests. 


\section{References}

1. Rhodes, J.S.; Best, K.; Belknap, J.K.; Finn, D.A.; Crabbe, J.C. Evaluation of a simple model of ethanol drinking to intoxication in C57BL/6J mice. Physiol. Behav. 2005, 84, 53-63. [CrossRef] [PubMed]

2. Rhodes, J.S.; Ford, M.M.; Yu, C.H.; Brown, L.L.; Finn, D.A.; Garland, T.; Crabbe, J.C. Mouse inbred strain differences in ethanol drinking to intoxication. Genes, Brain Behav. 2007, 6, 1-18. [CrossRef] [PubMed]

3. Crabbe, J.C.; Spence, S.E.; Brown, L.L.; Metten, P. Alcohol preference drinking in a mouse line selectively bred for high drinking in the dark. Alcohol. 2011, 45, 427-440. [CrossRef] [PubMed]

4. Crabbe, J.C.; Metten, P.; Rhodes, J.S.; Yu, C.-H.; Brown, L.L.; Phillips, T.J.; Finn, D.A. A line of mice selected for high blood ethanol concentrations shows drinking in the dark to intoxication. Biol. Psychiatry. 2009, 65, 662-670. [CrossRef]

5. Crabbe, J.C.; Metten, P.; Belknap, J.K.; Spence, S.E.; Cameron, A.J.; Schlumbohm, J.P.; Huang, L.C.; Barkley-Levenson, A.M.; Ford, M.M.; Phillips, T.J. Progress in a replicated selection for elevated blood ethanol concentrations in HDID mice. Genes, Brain Behav. 2014, 13, 236-246. [CrossRef]

6. Barkley-Levenson, A.M.; Crabbe, J.C. High Drinking in the Dark Mice: A genetic model of drinking to intoxication. Alcohol 2014, 48, 217-223. [CrossRef]

7. Crabbe, J.C.; Metten, P.; Savarese, A.M.; Ozburn, A.R.; Schlumbohm, J.P.; Spence, S.E.; Hack, W.R. Ethanol Conditioned Taste Aversion in High Drinking in the Dark Mice. Brain Sci. 2019, 9, 2. [CrossRef]

8. Ferguson, L.B.; Ozburn, A.R.; Ponomarev, I.; Metten, P.; Reilly, M.; Crabbe, J.C.; Harris, R.A.; Mayfield, R.D. Genome-Wide Expression Profiles Drive Discovery of Novel Compounds that Reduce Binge Drinking in Mice. Neuropsychopharmacology 2018, 43, 1257-1266. [CrossRef]

9. Iancu, O.D.; Oberbeck, D.; Darakjian, P.; Metten, P.; McWeeney, S.; Crabbe, J.C.; Hitzemann, R. Selection for Drinking in the Dark Alters Brain Gene Coexpression Networks. Alcohol. Clin. Exp. Res. 2013, 37, 1295-1303. [CrossRef]

10. Hitzemann, R.; Oberbeck, D.; Iancu, O.; Darakjian, P.; McWeeney, S.; Spence, S.; Schlumbohm, J.; Metten, P.; Crabbe, J. Alignment of the transcriptome with individual variation in animals selectively bred for High Drinking-In-the-Dark (HDID). Alcohol 2017, 60, 115-120. [CrossRef]

11. Barkley-Levenson, A.M.; Crabbe, J.C. Distinct ethanol drinking microstructures in two replicate lines of mice selected for drinking to intoxication. Genes, Brain Behav. 2015, 14, 398-410. [CrossRef] [PubMed]

12. Barkley-Levenson, A.M.; Crabbe, J.C. Genotypic and sex differences in anxiety-like behavior and alcohol-induced anxiolysis in High Drinking in the Dark selected mice. Alcohol 2015, 49, 29-36. [CrossRef] [PubMed]

13. Barkley-Levenson, A.M.; Cunningham, C.L.; Smitasin, P.J.; Crabbe, J.C. Rewarding and aversive effects of ethanol in High Drinking in the Dark selectively bred mice. Addict. Biol. 2015, 20, 80-90. [CrossRef]

14. McCulley, W.D.; Ascheid, S.; Crabbe, J.C.; Rosenwasser, A.M. Selective breeding for ethanol-related traits alters circadian phenotype. Alcohol 2013, 47, 187-194. [CrossRef] [PubMed]

15. Kamdar, N.K.; Miller, S.A.; Syed, Y.M.; Bhayana, R.; Gupta, T.; Rhodes, J.S. Acute effects of naltrexone and GBR 12909 on ethanol drinking-in-the-dark in C57BL/6J mice. Psychopharmacology (Berl.) 2007, 192, 207-217. [CrossRef]

16. Ozburn, A.R.; Harris, R.A.; Blednov, Y.A. Chronic voluntary alcohol consumption results in tolerance to sedative/hypnotic and hypothermic effects of alcohol in hybrid mice. Pharmacol. Biochem. Behav. 2013, 104, 33-39. [CrossRef]

17. Kasten, C.R.; Blasingame, S.N.; Boehm, S.L. Bidirectional enantioselective effects of the GABAB receptor agonist baclofen in two mouse models of excessive ethanol consumption. Alcohol 2015, 49, 37-46. [CrossRef]

18. Navarro, M.; Carvajal, F.; Lerma-Cabrera, J.M.; Cubero, I.; Picker, M.J.; Thiele, T.E. Evidence that Melanocortin Receptor Agonist Melanotan-II Synergistically Augments the Ability of Naltrexone to Blunt Binge-Like Ethanol Intake in Male C57BL/6J Mice. Alcohol. Clin. Exp. Res. 2015, 39, 1425-1433. [CrossRef]

19. Crabbe, J.C.; Ozburn, A.R.; Metten, P.; Barkley-Levenson, A.; Schlumbohm, J.P.; Spence, S.E.; Hack, W.R.; Huang, L.C. High Drinking in the Dark (HDID) mice are sensitive to the effects of some clinically relevant drugs to reduce binge-like drinking. Pharmacol. Biochem. Behav. 2017, 160, 55-62. [CrossRef]

20. Zhou, F.C.; Anthony, B.; Dunn, K.W.; Lindquist, W.B.; Xu, Z.C.; Deng, P. Chronic alcohol drinking alters neuronal dendritic spines in the brain reward center nucleus accumbens. Brain Res. 2007, 1134, 148-161. [CrossRef] 
21. Koob, G.F.; Volkow, N.D. Neurocircuitry of Addiction. Neuropsychopharmacology 2010, 35, 217-238. [CrossRef]

22. Heinze, H.-J.; Heldmann, M.; Voges, J.; Hinrichs, H.; Marco-Pallares, J.; Hopf, J.-M.; Müller, U.J.; Galazky, I.; Sturm, V.; Bogerts, B.; et al. Counteracting incentive sensitization in severe alcohol dependence using deep brain stimulation of the Nucleus accumbens: clinical and basic science aspects. Front. Hum. Neurosci. 2009, 3, 22. [CrossRef] [PubMed]

23. Kuhn, J.; Gründler, T.O.J.; Bauer, R.; Huff, W.; Fischer, A.G.; Lenartz, D.; Maarouf, M.; Bührle, C.; Klosterkötter, J.; Ullsperger, M.; et al. Successful deep brain stimulation of the nucleus accumbens in severe alcohol dependence is associated with changed performance monitoring. Addict. Biol. 2011, 16, 620-623. [CrossRef] [PubMed]

24. Voges, J.; Müller, U.; Bogerts, B.; Münte, T.; Heinze, H.-J. Deep Brain Stimulation Surgery for Alcohol Addiction. World Neurosurg. 2013, 80, S28.e21-S28.e31. [CrossRef] [PubMed]

25. Knapp, C.M.; Tozier, L.; Pak, A.; Ciraulo, D.A.; Kornetsky, C. Deep brain stimulation of the nucleus accumbens reduces ethanol consumption in rats. Pharmacol. Biochem. Behav. 2009, 92, 474-479. [CrossRef]

26. Henderson, M.B.; Green, A.I.; Bradford, P.S.; Chau, D.T.; Roberts, D.W.; Leiter, J.C. Deep brain stimulation of the nucleus accumbens reduces alcohol intake in alcohol-preferring rats. Neurosurg. Focus 2010, 29, E12. [CrossRef]

27. Ho, A.L.; Salib, A.-M.N.; Pendharkar, A.V.; Sussman, E.S.; Giardino, W.J.; Halpern, C.H. The nucleus accumbens and alcoholism: a target for deep brain stimulation. Neurosurg. Focus 2018, 45, E12. [CrossRef]

28. Roth, B.L. DREADDs for Neuroscientists. Neuron 2016, 89, 683-694. [CrossRef]

29. Purohit, K.; Parekh, P.K.; Kern, J.; Logan, R.W.; Liu, Z.; Huang, Y.; McClung, C.A.; Crabbe, J.C.; Ozburn, A.R. Pharmacogenetic Manipulation of the Nucleus Accumbens Alters Binge-Like Alcohol Drinking in Mice. Alcohol. Clin. Exp. Res. 2018, 42, 879-888. [CrossRef]

30. Bayram-Weston, Z.; Olsen, E.; Harrison, D.J.; Dunnett, S.B.; Brooks, S.P. Optimising Golgi-Cox staining for use with perfusion-fixed brain tissue validated in the zQ175 mouse model of Huntington's disease. J. Neurosci. Methods 2016, 265, 81-88. [CrossRef]

31. Aarts, E.; Verhage, M.; Veenvliet, J.V.; Dolan, C.V.; van der Sluis, S. A solution to dependency: using multilevel analysis to accommodate nested data. Nat. Neurosci. 2014, 17, 491-496. [CrossRef] [PubMed]

32. Bates, D.; Mächler, M.; Bolker, B.; Walker, S. Fitting Linear Mixed-Effects Models Using lme4. J. Stat. Software 2015. [CrossRef]

33. Benjamini, Y.; Hochberg, Y. Controlling the False Discovery Rate: A Practical and Powerful Approach to Multiple Testing. J. R. Stat. Soc. Ser. B 1995, 57, 289-300. [CrossRef]

34. FastQC: A Quality Control Tool for High Throughput Sequence Data. Available online: http://www. bioinformatics.babraham.ac.uk?/projects/fastqc/ (accessed on 10 February 2020).

35. Dobin, A.; Davis, C.A.; Schlesinger, F.; Drenkow, J.; Zaleski, C.; Jha, S.; Batut, P.; Chaisson, M.; Gingeras, T.R. STAR: ultrafast universal RNA-seq aligner. Bioinformatics 2013, 29, 15-21. [CrossRef]

36. Anders, S.; Pyl, P.T.; Huber, W. HTSeq-a Python framework to work with high-throughput sequencing data. Bioinformatics 2015, 31, 166-169. [CrossRef]

37. Development Core Team, R. R: A Language and Environment for Statistical Computing. Available online: http://www.r-project.org (accessed on 10 February 2020).

38. Law, C.W.; Chen, Y.; Shi, W.; Smyth, G.K. voom: precision weights unlock linear model analysis tools for RNA-seq read counts. Genome Biol. 2014, 15, R29. [CrossRef]

39. Hulsen, T.; de Vlieg, J.; Alkema, W. BioVenn - a web application for the comparison and visualization of biological lists using area-proportional Venn diagrams. BMC Genomics 2008, 9, 488. [CrossRef]

40. Russo, S.J.; Dietz, D.M.; Dumitriu, D.; Morrison, J.H.; Malenka, R.C.; Nestler, E.J. The addicted synapse: Mechanisms of synaptic and structural plasticity in nucleus accumbens. Trends Neurosci. 2010. [CrossRef]

41. Arikkath, J. Molecular mechanisms of dendrite morphogenesis. Front. Cell. Neurosci. 2012. [CrossRef]

42. Uys, J.D.; McGuier, N.S.; Gass, J.T.; Griffin, W.C.; Ball, L.E.; Mulholland, P.J. Chronic intermittent ethanol exposure and withdrawal leads to adaptations in nucleus accumbens core postsynaptic density proteome and dendritic spines. Addict. Biol. 2016. [CrossRef]

43. Langfelder, P.; Horvath, S. WGCNA: an R package for weighted correlation network analysis. BMC Bioinform. 2008, 9, 559. [CrossRef] [PubMed]

44. Tritchler, D.; Parkhomenko, E.; Beyene, J. Filtering Genes for Cluster and Network Analysis. BMC Bioinform. 2009, 10, 193. [CrossRef] [PubMed] 
45. Warde-Farley, D.; Donaldson, S.L.; Comes, O.; Zuberi, K.; Badrawi, R.; Chao, P.; Franz, M.; Grouios, C.; Kazi, F.; Lopes, C.T.; et al. The GeneMANIA prediction server: biological network integration for gene prioritization and predicting gene function. Nucleic Acids Res. 2010, 38, W214-W220. [CrossRef] [PubMed]

46. Eden, E.; Navon, R.; Steinfeld, I.; Lipson, D.; Yakhini, Z. GOrilla: a tool for discovery and visualization of enriched GO terms in ranked gene lists. BMC Bioinformatics 2009, 10, 48. [CrossRef] [PubMed]

47. Kuleshov, M.V.; Jones, M.R.; Rouillard, A.D.; Fernandez, N.F.; Duan, Q.; Wang, Z.; Koplev, S.; Jenkins, S.L.; Jagodnik, K.M.; Lachmann, A.; et al. Enrichr: a comprehensive gene set enrichment analysis web server 2016 update. Nucleic Acids Res. 2016, 44, W90-W97. [CrossRef]

48. Akbar, M.; Egli, M.; Cho, Y.-E.; Song, B.-J.; Noronha, A. Medications for alcohol use disorders: An overview. Pharmacol. Ther. 2018, 185, 64-85. [CrossRef]

49. Williams, E.C.; Matson, T.E.; Harris, A.H.S. Strategies to increase implementation of pharmacotherapy for alcohol use disorders: a structured review of care delivery and implementation interventions. Addict. Sci. Clin. Pract. 2019, 14, 6. [CrossRef]

50. Cassataro, D.; Bergfeldt, D.; Malekian, C.; Van Snellenberg, J.X.; Thanos, P.K.; Fishell, G.; Sjulson, L. Reverse pharmacogenetic modulation of the nucleus accumbens reduces ethanol consumption in a limited access paradigm. Neuropsychopharmacology 2014, 39, 283-290. [CrossRef]

51. DePoy, L.; Daut, R.; Brigman, J.L.; MacPherson, K.; Crowley, N.; Gunduz-Cinar, O.; Pickens, C.L.; Cinar, R.; Saksida, L.M.; Kunos, G.; et al. Chronic alcohol produces neuroadaptations to prime dorsal striatal learning. Proc. Natl. Acad. Sci. USA 2013, 110, 14783-14788. [CrossRef]

52. Wang, J.; Cheng, Y.; Wang, X.; Roltsch Hellard, E.; Ma, T.; Gil, H.; Ben Hamida, S.; Ron, D. Alcohol Elicits Functional and Structural Plasticity Selectively in Dopamine D1 Receptor-Expressing Neurons of the Dorsomedial Striatum. J. Neurosci. 2015, 35, 11634-11643. [CrossRef]

53. Kupchik, Y.M.; Kalivas, P.W. The Direct and Indirect Pathways of the Nucleus Accumbens are not What You Think. Neuropsychopharmacology 2017, 42, 369-370. [CrossRef] [PubMed]

54. Moonat, S.; Starkman, B.G.; Sakharkar, A.; Pandey, S.C. Neuroscience of alcoholism: molecular and cellular mechanisms. Cell. Mol. Life Sci. 2010, 67, 73-88. [CrossRef] [PubMed]

55. Spijker, S.; Houtzager, S.W.J.; De Gunst, M.C.M.; De Boer, W.P.H.; Schoffelmeer, A.N.M.; Smit, A.B. Morphine exposure and abstinence define specific stages of gene expression in the rat nucleus accumbens. FASEB J. 2004, 18, 848-850. [CrossRef] [PubMed]

56. Roche, K.W. The expanding role of PSD-95: a new link to addiction. Trends Neurosci. 2004, 27, 699-700. [CrossRef] [PubMed]

57. Yao, W.-D.; Gainetdinov, R.R.; Arbuckle, M.I.; Sotnikova, T.D.; Cyr, M.; Beaulieu, J.-M.; Torres, G.E.; Grant, S.G.N.; Caron, M.G. Identification of PSD-95 as a regulator of dopamine-mediated synaptic and behavioral plasticity. Neuron 2004, 41, 625-638. [CrossRef]

58. Mulligan, M.K.; Rhodes, J.S.; Crabbe, J.C.; Mayfield, R.D.; Adron Harris, R.; Ponomarev, I. Molecular Profiles of Drinking Alcohol to Intoxication in C57BL/6J Mice. Alcohol. Clin. Exp. Res. 2011, 35, 659-670. [CrossRef]

59. Ferguson, L.B.; Zhang, L.; Kircher, D.; Wang, S.; Mayfield, R.D.; Crabbe, J.C.; Morrisett, R.A.; Harris, R.A.; Ponomarev, I. Dissecting Brain Networks Underlying Alcohol Binge Drinking Using a Systems Genomics Approach. Mol. Neurobiol. 2019, 56, 2791-2810.

60. Ozburn, A.R.; Falcon, E.; Mukherjee, S.; Gillman, A.; Arey, R.; Spencer, S.; McClung, C.A. The Role of Clock in Ethanol-Related Behaviors. Neuropsychopharmacology 2013, 38, 2393-2400. [CrossRef]

61. Blednov, Y.A.; Black, M.; Chernis, J.; Da Costa, A.; Mayfield, J.; Harris, R.A. Ethanol Consumption in Mice Lacking CD14, TLR2, TLR4, or MyD88. Alcohol. Clin. Exp. Res. 2017, 41, 516-530. [CrossRef]

62. Wolstenholme, J.T.; Warner, J.A.; Capparuccini, M.I.; Archer, K.J.; Shelton, K.L.; Miles, M.F. Genomic analysis of individual differences in ethanol drinking: evidence for non-genetic factors in C57BL/6 mice. PLoS ONE 2011, 6, e21100. [CrossRef]

63. Sakharkar, A.J.; Zhang, H.; Tang, L.; Shi, G.; Pandey, S.C. Histone Deacetylases (HDAC)-Induced Histone Modifications in the Amygdala: A Role in Rapid Tolerance to the Anxiolytic Effects of Ethanol. Alcohol. Clin. Exp. Res. 2012, 36, 61-71. [CrossRef] [PubMed]

64. Simon-O'Brien, E.; Alaux-Cantin, S.; Warnault, V.; Buttolo, R.; Naassila, M.; Vilpoux, C. The histone deacetylase inhibitor sodium butyrate decreases excessive ethanol intake in dependent animals. Addict. Biol. 2015, 20, 676-689. [CrossRef] [PubMed] 
65. Trazzi, S.; Fuchs, C.; Viggiano, R.; De Franceschi, M.; Valli, E.; Jedynak, P.; Hansen, F.K.; Perini, G.; Rimondini, R.; Kurz, T.; et al. HDAC4: a key factor underlying brain developmental alterations in CDKL5 disorder. Hum. Mol. Genet. 2016, 25, 3887-3907. [CrossRef] [PubMed]

66. Bourgeron, T. From the genetic architecture to synaptic plasticity in autism spectrum disorder. Nat. Rev. Neurosci. 2015, 16, 551-563. [CrossRef]

(C) 2020 by the authors. Licensee MDPI, Basel, Switzerland. This article is an open access article distributed under the terms and conditions of the Creative Commons Attribution (CC BY) license (http://creativecommons.org/licenses/by/4.0/). 